\title{
A Stochastic HIV Infection Model with Latent Infection and Antiretroviral Therapy
}

\author{
Jun Liu $(\mathbb{D}),{ }^{1}$ Yan Wang $\mathbb{D}^{1},{ }^{1}$ Luju Liu $\mathbb{D}^{\mathrm{D}},{ }^{2}$ and Tingting Zhao $\mathbb{D}^{3}$ \\ ${ }^{1}$ College of Science, China University of Petroleum (East China), Qingdao, Shandong 266580, China \\ ${ }^{2}$ School of Mathematics and Statistics, Henan University of Science and Technology, Luoyang, Henan 471023, China \\ ${ }^{3}$ School of Mathematics, Northwest University, Xian, Shaanxi 710127, China
}

Correspondence should be addressed to Yan Wang; wangyan@upc.edu.cn

Received 2 February 2018; Accepted 12 April 2018; Published 25 June 2018

Academic Editor: Vicenç Méndez

Copyright (C) 2018 Jun Liu et al. This is an open access article distributed under the Creative Commons Attribution License, which permits unrestricted use, distribution, and reproduction in any medium, provided the original work is properly cited.

\begin{abstract}
Recent studies have demonstrated that the latent infection is a major obstacle to the viral elimination in HIV infection process. In this paper, we formulate a stochastic HIV infection model to include both latent infection and combination drug therapies. We derive that the model solution is unique and positive, and the solution is global. By constructing appropriate stochastic Lyapunov functions, the existence of an ergodic stationary distribution is obtained when the critical condition is greater than one. Furthermore, through rigorous analysis and deduction, the extinction of the virus is established under certain conditions. Numerical simulations are performed to show that small intensity of white noises can maintain the existence of a stationary distribution, while large intensity of white noises is beneficial to the extinction of the virus.
\end{abstract}

\section{Introduction}

More and more mathematical models have been developed to reflect the dynamics mechanism of HIV virus. The classical HIV virus model is based on three-dimensional ordinary differential equations, which contains the target T-cells population, infected T-cells population, and virions [1-3]. With the progress of HIV drug treatment, some researchers have investigated the effects of drug therapies on model behaviors [4-7]. The commonly used highly active antiretroviral therapy (HAART) is combined by reverse transcriptase inhibitors and protease inhibitors. Reverse transcriptase inhibitors can inhibit the activity of reverse transcriptase and prevent the formation of provirus during the virus infection; protease inhibitors can block the virus infection of new target T-cells. Unfortunately, the current drug treatment can not eradicate the virus thoroughly. With the development of cell and molecular biology, it has been discovered that the existence of latent infection is a major obstacle to clear the virus $[8,9]$.

In recent years, mathematical models with the inclusion of latent infected T-cells have been developed to investigate the model behaviors [10-15]. However, all these models are deterministic model, and the effect of stochastic fluctuation factor is not considered. Actually, HIV transcription is an inherent random process and produces strong random fluctuations in the HIV gene products [16-18]. Thus, by experimental data, it has been proved that these random factors seriously affect the evolution of HIV virus during the protease inhibitor therapy [16]. Moreover, Weinberger et al. have demonstrated that stochastic fluctuation could play an important role in delaying HIV transactivation and contributing to latency [19]. Therefore, it is necessary to consider stochastic fluctuation in HIV virus model, which will be more appropriate to reflect the virus infection process.

Recently, the stochastic differential equation models of infectious diseases [20-25] have been greatly developed, but the stochastic differential equation model of the virus dynamic model has just been developed. The classic threedimensional stochastic model is studied in the earlier literatures [26-29], then the virus stochastic model with target T-cell logistic growth and CTL immune response is also developed in the last two years [30,31]. However, all these models did not consider the latent infection mechanism. Conway and Coombs have formulated a stochastic model of latent infected T-cells [32], but they neither include the target T-cell logistic growth nor analyze the model dynamics 
theoretically. In this paper, we will formulate an HIV model with latent infection, healthy T-cell logistic growth, antiretroviral therapy, and random perturbations.

The organization of this paper is as follows. In Section 2, we construct our stochastic model and give the existence and uniqueness of the global positive solution. By constructing suitable stochastic Lyapunov functions, the existence of a unique ergodic stationary distribution is derived in Section 3. In Section 4, the sufficient conditions for the extinction of the virus are obtained, and numerical simulations are also employed to show how the white noises affect the model behaviors in Section 5. We summarize our results and give future work finally.

\section{Model and Preliminaries}

For deterministic model, Wang et al. [14] have studied the following HIV model with latent infection, T-cell logistic growth, and antiretroviral therapy,

$$
\begin{aligned}
& \frac{d}{d t} T(t)=\lambda-d_{T} T+r T\left(1-\frac{T}{T_{\max }}\right)-\left(1-n_{r t}\right) k V T, \\
& \frac{d}{d t} L(t)=\eta\left(1-n_{r t}\right) k V T-d_{L} L-a L \\
& \frac{d}{d t} I(t)=(1-\eta)\left(1-n_{r t}\right) k V T-\delta I+a L, \\
& \frac{d}{d t} V(t)=\left(1-n_{p}\right) N \delta T^{*}-c V .
\end{aligned}
$$

Here, $T(t), L(t)$, and $I(t)$ represent the concentrations of healthy T-cells, latent infected T-cells, and actively infected T-cells at time $t$, respectively. $V(\mathrm{t})$ denote the infectious viral particles at time $t$. Parameter $\lambda$ is a constant input rate, $r$ is the logistic growth rate, and $T_{\max }$ is the maximum capacity of the number of healthy T-cells in the blood. $k$ is the infection rate between healthy T-cells and infectious virus, $\eta$ is the fraction of infections leading to latency, and $a$ is the activated rate. Parameters $d_{T}, d_{L}, \delta$, and $c$ are the death rates of the healthy T-cells, latently infected T-cells, actively infected Tcells, and infectious virions, respectively. $N$ is the burst size of one productively infected T-cell. $n_{r t}$ and $n_{p}$ denote the drug efficacy of reverse transcriptase inhibitors and protease inhibitors $\left(0 \leq n_{r t}<1\right.$ and $\left.0 \leq n_{p}<1\right)$, respectively.

Wang et al. in literature [14] have analyzed the theoretical results of system (1). System (1) always has an uninfected equilibrium $E_{0}\left(T_{0}, 0,0,0\right)$, where

$$
T_{0}=\frac{T_{\max }}{2 r}\left[r-d_{T}+\sqrt{\left(r-d_{T}\right)^{2}+\frac{4 r \lambda}{T_{\max }}}\right],
$$

and the basic reproduction number is

$$
\mathscr{R}_{0}=\frac{k N T_{0}\left(1-n_{r t}\right)\left(1-n_{p}\right)\left[a+(1-\eta) d_{L}\right]}{c\left(a+d_{L}\right)} .
$$

When $\mathscr{R}_{0}>1$, only one infected equilibrium $\bar{E}(\bar{T}, \bar{L}, \bar{I}, \bar{V})$ exists. Wang et al. have obtained the following theoretical results of system (1):
(I) If $\mathscr{R}_{0}<1$, the uninfected equilibrium $E_{0}$ is globally asymptotically stable.

(II) If $\mathscr{R}_{0}>1$, then the infected equilibrium $\bar{E}$ is locally asymptotically stable under certain condition, and the uninfected equilibrium $E_{0}$ is unstable.

In this paper, considering random fluctuation during HIV infection process [16-19], we follow the assumptions of previous literatures [26-31] and assume that the parameters $-d_{T},-d_{L},-\delta$, and $-c$ are perturbed by the white noise type. In other words, $-d_{T} \rightarrow-d_{T}+\sigma_{1} \dot{B}_{1}(t),-d_{L} \rightarrow-d_{L}+$ $\sigma_{2} \dot{B}_{2}(t),-\delta \rightarrow-\delta+\sigma_{3} \dot{B}_{3}(t)$, and $-c \rightarrow-c+\sigma_{4} \dot{B}_{4}(t)$. Thus,

$$
\begin{aligned}
-d_{T} d t & \longrightarrow-d_{T} d t+\sigma_{1} d B_{1}(t), \\
-d_{L} d t & \longrightarrow-d_{L} d t+\sigma_{2} d B_{2}(t), \\
-\delta d t & \longrightarrow-\delta d t+\sigma_{3} d B_{3}(t), \\
-c d t & \longrightarrow-c d t+\sigma_{4} d B_{4}(t),
\end{aligned}
$$

where $B_{i}(t)$ are independent standard Brownian motions with $B_{i}(0)=0$ and $\sigma_{i}^{2}>0$ denote the intensities of the white noise, $i=1,2,3,4$. Then, system (1) with white noises can be written as

$$
\begin{aligned}
& d T(t) \\
& =\left[\lambda-d_{T} T+r T\left(1-\frac{T}{T_{\max }}\right)-\left(1-n_{r t}\right) k V T\right] d t \\
& \quad+\sigma_{1} T d B_{1}(t),
\end{aligned}
$$

$d L(t)$

$$
=\left[\eta\left(1-n_{r t}\right) k V T-d_{L} L-a L\right] d t+\sigma_{2} L d B_{2}(t),
$$

$d I(t)$

$$
\begin{aligned}
= & {\left[(1-\eta)\left(1-n_{r t}\right) k V T-\delta I+a L\right] d t } \\
& +\sigma_{3} I d B_{3}(t), \\
d V(t) & =\left[\left(1-n_{p}\right) N \delta I-c V\right] d t+\sigma_{4} V d B_{4}(t) .
\end{aligned}
$$

In the following of this paper, we assume $\left(\Omega, \mathscr{F},\left\{\mathscr{F}_{t}\right\}_{t \geq 0}\right.$, P) be a complete probability space with a filtration $\left\{\mathscr{F}_{t}\right\}_{t \geq 0}$ satisfying the usual conditions (i.e., it is increasing and right continuous while $\mathscr{F}_{0}$ contains all $\mathbb{P}$-null sets $)$, and $B_{i}(t)(i=$ $1,2,3,4)$ are defined on this complete probability space. We also let $\mathbb{R}_{+}^{4}=\left\{x=\left(x_{1}, x_{2}, x_{3}, x_{4}\right) \in \mathbb{R}^{4}: x_{i}>0, i=1,2,3,4\right\}$.

For convenience, we use symbol $a \wedge b$ to represent $\min \{a, b\}$ and use symbol $a \vee b$ to represent $\max \{a, b\}$. We further denote the following notations:

$$
\begin{aligned}
\bar{k} & :=\left(1-n_{r t}\right) k, \\
\bar{N} & :=\left(1-n_{p}\right) N .
\end{aligned}
$$

Based on the theory of Mao [33] and the article of Mao et al. [34], we show the existence and uniqueness of the solution of system (5) in the following theorem. 
Theorem 1. For any initial value $(T(0), L(0), I(0), V(0)) \in \mathbb{R}_{+}^{4}$ in system (5), there exists a unique positive solution $(T(t), L(t)$, $I(t), V(t))$ and the solution will be contained in $\mathbb{R}_{+}^{4}$ with probability one, namely, $(T(t), L(t), I(t), V(t)) \in \mathbb{R}_{+}^{4}$ for all $t \geq 0$ almost surely (a.s.).

Proof. All the coefficients of system (5) are locally Lipschitz continuous, by the theory of stochastic differential equation [33], so we obtain that there exists a unique maximum local solution $(T(t), L(t), I(t), V(t))$ on $t \in\left[0, \tau_{e}\right)$, where $\tau_{e}$ is the explosion time.

We show the solution is global in the following; namely, we should derive that $\tau_{e}=\infty$ a.s. Otherwise, it is supposed that there is a finite time so that the solution $(T(t), L(t), I(t), V(t))$ can not explode to infinity. Let $n_{0}>0$ be big enough so that $T(0), L(0), I(0)$ and $V(0)$ are all on the interval $\left[1 / n_{0}, n_{0}\right]$. For each integer $n \geq n_{0}$, the stopping time is defined as follows:

$$
\begin{aligned}
\tau_{n} & =\inf \left\{t \in\left[0, \tau_{e}\right): \min \{T(t), L(t), I(t), V(t)\}\right. \\
& \left.\leq \frac{1}{n} \text { or } \max \{T(t), L(t), I(t), V(t)\} \geq n\right\},
\end{aligned}
$$

and we let $\inf \emptyset=\infty$ (commonly, $\emptyset=$ the empty set). It is obvious that $\tau_{n}$ increases with $n \rightarrow \infty$. We denote $\tau_{\infty}=$ $\lim _{n \rightarrow \infty} \tau_{n}$, then $\tau_{\infty} \leq \tau_{e}$ a.s.

(i) If $\tau_{\infty}=\infty$ is satisfied a.s., then $\tau_{e}=\infty$ a.s., so $(T(t), L(t), I(t), V(t)) \in \mathbb{R}_{+}^{4}$ a.s. for all $t \geq 0$.

(ii) Otherwise, we assume that there exists a pair of constants $\tilde{t}>0$ and $\epsilon \in(0,1)$ such that $\mathbb{P}\left\{\tau_{\infty} \leq \widetilde{t}\right\}>\epsilon$. So, there exists an integer $n_{1} \geq n_{0}$ that makes $\mathbb{P}\left\{\tau_{\infty} \leq \widetilde{t}\right\} \geq$ $\epsilon$ for all $n \geq n_{1}$ valid.

Define a $C^{2}$-function $W: \mathbb{R}_{+}^{4} \rightarrow \mathbb{R}_{+}$,

$$
\begin{aligned}
W(T, L, I, V)= & \left(T-b-b \ln \frac{T}{b}\right)+(L-1-\ln L) \\
& +(I-1-\ln I)+\frac{1}{2 \bar{N}}(V-1-\ln V),
\end{aligned}
$$

where $b$ is a positive constant to be determined. Employing Itô's formula [33] to $W$ yields

$$
\begin{aligned}
d W(T, L, I, V)= & L W(T, L, I, V) d t \\
& +\sigma_{1}(T-b) d B_{1}(t) \\
& +\sigma_{2}(L-1) d B_{2}(t) \\
& +\sigma_{3}(I-1) d B_{3}(t) \\
& +\frac{\sigma_{4}}{2 \bar{N}}(V-1) d B_{4}(t),
\end{aligned}
$$

$$
\mathscr{R}_{0}^{s}=\frac{\lambda \overline{k \bar{N}} \delta\left[a+(1-\eta)\left(d_{L}+(1 / 2) \sigma_{2}^{2}\right)\right]}{\left(\lambda / T_{0}+(1 / 2) \sigma_{1}^{2}\right)\left(d_{L}+a+(1 / 2) \sigma_{2}^{2}\right)\left(\delta+(1 / 2) \sigma_{3}^{2}\right)\left(c+(1 / 2) \sigma_{4}^{2}\right)},
$$

and denote $\sigma^{2}=\sigma_{1}^{2} \vee \sigma_{2}^{2} \vee \sigma_{3}^{2} \vee \sigma_{4}^{2}$. 
Theorem 2. When $\mathscr{R}_{0}^{s}>1$, system (5) has a unique ergodic stationary distribution $\pi(\cdot)$.

Proof. According to Lemma A.1 in the Appendix, we divide it into two steps to prove our conclusion. Now, we prove the validity of condition (A1) in Lemma A.1. The diffusion matrix of system (5) is

$$
A=\left[\begin{array}{cccc}
\sigma_{1}^{2} T^{2} & 0 & 0 & 0 \\
0 & \sigma_{2}^{2} L^{2} & 0 & 0 \\
0 & 0 & \sigma_{3}^{2} I^{2} & 0 \\
0 & 0 & 0 & \sigma_{4}^{2} V^{2}
\end{array}\right] .
$$

Choose $R=\min _{(T, L, I, V) \in \bar{D}_{l} \subset \mathbb{R}_{+}^{4}}\left\{\sigma_{1}^{2} T^{2}, \sigma_{2}^{2} L^{2}, \sigma_{3}^{2} I^{2}, \sigma_{4}^{2} V^{2}\right\}$; we have

$$
\begin{aligned}
& \sum_{i, j=1}^{4} a_{i j}(T, L, I, V) \xi_{i} \xi_{j}= \sigma_{1}^{2} T^{2} \xi_{1}^{2}+\sigma_{2}^{2} L^{2} \xi_{2}^{2}+\sigma_{3}^{2} I^{2} \xi_{3}^{2} \\
&+\sigma_{4}^{2} V^{2} \xi_{4}^{2} \geq \sigma^{2}|\xi|^{2}, \\
&(T, L, I, V) \in \bar{D}_{s}, \xi=\left(\xi_{1}, \xi_{2}, \xi_{3}, \xi_{4}\right) \in \mathbb{R}_{+}^{4},
\end{aligned}
$$

where $\bar{D}_{l}=[1 / l, 1] \times[1 / l, l] \times[1 / l, l] \times[1 / l, l]$ and $l$ is a sufficiently large integer, then condition (A1) in Lemma A.1 is satisfied.

In the following, we illustrate the establishment of condition (A2) in Lemma A.1 in the Appendix. Denote

$$
f(T)=\lambda+\left(r-d_{T}\right) T-\frac{r}{T_{\max }} T^{2},
$$

and $f$ is a quadratic function with respect to $T$. Suppose $T_{0}$ and $-T_{1}$ are the two roots of $f(T)=0$, then

$$
f(T)=-\frac{r}{T_{\max }}\left(T-T_{0}\right)\left(T+T_{1}\right) .
$$

Since $-\left(T-T_{0}\right)^{2}=-\left(T-T_{0}\right)\left(T+T_{1}\right)+\left(T-T_{0}\right)\left(T_{0}+T_{1}\right) \leq 0$, then $\left(T-T_{0}\right)\left(T+T_{1}\right) \geq\left(T-T_{0}\right)\left(T_{0}+T_{1}\right)$, and

$$
\begin{aligned}
f(T) & =-\frac{r}{T_{\max }}\left(T-T_{0}\right)\left(T+T_{1}\right) \\
& \leq-\frac{r}{T_{\max }}\left(T-T_{0}\right)\left(T_{0}+T_{1}\right) .
\end{aligned}
$$

We define a $C^{2}$-function $W_{1}: \mathbb{R}_{+}^{4} \rightarrow \mathbb{R}$

$$
W_{1}=\frac{T}{T_{0}+T_{1}}-\ln T \text {, }
$$

and then,

$$
\begin{aligned}
L W_{1}= & \frac{1}{T_{0}+T_{1}}(f(T)-k V T)-\frac{\lambda}{T}+d_{T} \\
& -r\left(1-\frac{T}{T_{\max }}\right)+\bar{k} V+\frac{1}{2} \sigma_{1}^{2} \\
\leq & \frac{f(T)}{T_{0}+T_{1}}-\frac{\lambda}{T}+d_{T}-r+\frac{r T}{T_{\max }}+\bar{k} V+\frac{1}{2} \sigma_{1}^{2} \\
\leq & -\frac{r}{T_{\max }}\left(T-T_{0}\right)-\frac{\lambda}{T}+d_{T}-r+\frac{r T}{T_{\max }}+\bar{k} V \\
& +\frac{1}{2} \sigma_{1}^{2} \leq-\frac{\lambda}{T}+\frac{\lambda}{T_{0}}+\bar{k} V+\frac{1}{2} \sigma_{1}^{2} .
\end{aligned}
$$

Here, we use the equality $d_{T}-r+r T_{0} / T_{\max }=\lambda / T_{0}$.

We construct a $C^{2}$-function $U_{1}: \mathbb{R}_{+}^{4} \rightarrow \mathbb{R}$,

$$
\begin{aligned}
U_{1}= & -\ln I+\left(c_{1}+d_{1}\right)\left(\frac{T}{T_{0}+T_{1}}-\ln T\right) \\
& -\left(c_{2}+d_{2}\right) \ln V-d_{3} \ln L,
\end{aligned}
$$

where $c_{1}, c_{2}, d_{1}, d_{2}$, and $d_{3}$ are positive constants which will be confirmed later. Applying Itô's formula [33] to $U_{1}$ and using inequality (19) yield

$$
\begin{aligned}
L U_{1} \leq & -\left[\frac{(1-\eta) \bar{k} T V}{I}+c_{1} \frac{\lambda}{T}+c_{2} \frac{\bar{N} \delta I}{V}\right] \\
& -\left(\frac{a L}{I}+d_{1} \frac{\lambda}{T}+d_{2} \frac{\bar{N} \delta I}{V}+d_{3} \frac{\eta \bar{k} V T}{L}\right) \\
& +\left(c_{1}+d_{1}\right)\left(\frac{\lambda}{T_{0}}+\frac{1}{2} \sigma_{1}^{2}+\bar{k} V\right) \\
& +\left(c_{2}+d_{2}\right)\left(c+\frac{1}{2} \sigma_{4}^{2}\right)+d_{3}\left(d_{L}+a+\frac{1}{2} \sigma_{2}^{2}\right) \\
& +\left(\delta+\frac{1}{2} \sigma_{3}^{2}\right) \\
\leq & -3 \sqrt[3]{c_{1} c_{2}(1-\eta) \lambda \bar{k} \bar{N} \delta}+c_{1}\left(\frac{\lambda}{T_{0}}+\frac{1}{2} \sigma_{1}^{2}\right) \\
& +c_{2}\left(c+\frac{1}{2} \sigma_{4}^{2}\right)-4 \sqrt[4]{d_{1} d_{2} d_{3} a \lambda \eta \bar{k} \bar{N} \delta} \\
& +d_{1}\left(\frac{\lambda}{T_{0}}+\frac{1}{2} \sigma_{1}^{2}\right)+d_{2}\left(c+\frac{1}{2} \sigma_{4}^{2}\right) \\
& +d_{3}\left(d_{L}+a+\frac{1}{2} \sigma_{2}^{2}\right)+\left(\delta+\frac{1}{2} \sigma_{3}^{2}\right) \\
& +\left(c_{1}+d_{1}\right) \bar{k} V .
\end{aligned}
$$


Let

$$
\begin{aligned}
& c_{1}\left(\frac{\lambda}{T_{0}}+\frac{1}{2} \sigma_{1}^{2}\right)=c_{2}\left(c+\frac{1}{2} \sigma_{4}^{2}\right) \\
& =\frac{(1-\eta) \lambda \bar{k} \bar{N} \delta}{\left(\lambda / T_{0}+(1 / 2) \sigma_{1}^{2}\right)\left(c+(1 / 2) \sigma_{4}^{2}\right)}, \\
& d_{1}\left(\frac{\lambda}{T_{0}}+\frac{1}{2} \sigma_{1}^{2}\right)=d_{2}\left(c+\frac{1}{2} \sigma_{4}^{2}\right)=d_{3}\left(d_{L}+a+\frac{1}{2} \sigma_{2}^{2}\right) \\
& =\frac{a \lambda \eta \bar{k} \bar{N} \delta}{\left(\lambda / T_{0}+(1 / 2) \sigma_{1}^{2}\right)\left(d_{L}+a+(1 / 2) \sigma_{2}^{2}\right)\left(c+(1 / 2) \sigma_{4}^{2}\right)} .
\end{aligned}
$$

We calculate that

$$
\begin{aligned}
& c_{1}=\frac{(1-\eta) \lambda \bar{k} \bar{N} \delta}{\left(\lambda / T_{0}+(1 / 2) \sigma_{1}^{2}\right)^{2}\left(c+(1 / 2) \sigma_{4}^{2}\right)}, \\
& c_{2}=\frac{(1-\eta) \lambda \bar{k} \bar{N} \delta}{\left(\lambda / T_{0}+(1 / 2) \sigma_{1}^{2}\right)\left(c+(1 / 2) \sigma_{4}^{2}\right)^{2}}, \\
& d_{1} \\
& =\frac{a \lambda \eta \bar{k} \bar{N} \delta}{\left(\lambda / T_{0}+(1 / 2) \sigma_{1}^{2}\right)^{2}\left(d_{L}+a+(1 / 2) \sigma_{2}^{2}\right)\left(c+(1 / 2) \sigma_{4}^{2}\right)}, \\
& d_{2} \\
& =\frac{a \lambda \eta \bar{k} \bar{N} \delta}{\left(\lambda / T_{0}+(1 / 2) \sigma_{1}^{2}\right)\left(d_{L}+a+(1 / 2) \sigma_{2}^{2}\right)\left(c+(1 / 2) \sigma_{4}^{2}\right)^{2}}, \\
& d_{3} \\
& =\frac{a \lambda \eta \bar{k} \bar{N} \delta}{\left(\lambda / T_{0}+(1 / 2) \sigma_{1}^{2}\right)\left(d_{L}+a+(1 / 2) \sigma_{2}^{2}\right)^{2}\left(c+(1 / 2) \sigma_{4}^{2}\right)} .
\end{aligned}
$$

Hence,

$$
\begin{aligned}
& L U_{1} \\
& \leq-\frac{(1-\eta) \lambda \bar{k} \bar{N} \delta}{\left(\lambda / T_{0}+(1 / 2) \sigma_{1}^{2}\right)\left(c+(1 / 2) \sigma_{4}^{2}\right)} \\
& \quad-\frac{a \lambda \eta \bar{k} \bar{N} \delta}{\left(\lambda / T_{0}+(1 / 2) \sigma_{1}^{2}\right)\left(d_{L}+a+(1 / 2) \sigma_{2}^{2}\right)\left(c+(1 / 2) \sigma_{4}^{2}\right)} \\
&+\left(\delta+\frac{1}{2} \sigma_{3}^{2}\right)+\left(c_{1}+d_{1}\right) \bar{k} V \\
&=-\frac{\lambda \bar{k} \bar{N} \delta\left[a+(1-\eta)\left(d_{L}+(1 / 2) \sigma_{2}^{2}\right)\right]}{\left(\lambda / T_{0}+(1 / 2) \sigma_{1}^{2}\right)\left(d_{L}+a+(1 / 2) \sigma_{2}^{2}\right)\left(c+(1 / 2) \sigma_{4}^{2}\right)} \\
&+\left(\delta+\frac{1}{2} \sigma_{3}^{2}\right)+\left(c_{1}+d_{1}\right) \bar{k} V \\
&=-\left(\delta+\frac{1}{2} \sigma_{3}^{2}\right)\left(\mathscr{R}_{0}^{s}-1\right)+\left(c_{1}+d_{1}\right) \bar{k} V:=-H+B V,
\end{aligned}
$$

where $\mathscr{R}_{0}^{s}$ is defined in (12) and

$$
\begin{aligned}
H & =\left(\delta+\frac{1}{2} \sigma_{3}^{2}\right)\left(\mathscr{R}_{0}^{s}-1\right), \\
B & =\left(c_{1}+d_{1}\right) \bar{k} .
\end{aligned}
$$

Define a $C^{2}$-function $U: \mathbb{R}_{+}^{4} \rightarrow \mathbb{R}$, in the following form:

$$
\begin{aligned}
U(T, L, I, V)= & M U_{1}-\ln T-\ln L-\ln I \\
& +\frac{1}{1+\theta}\left(T+L+I+\frac{V}{2 \bar{N}}\right)^{1+\theta} \\
:= & M U_{1}+U_{2}+U_{3}+U_{4}+U_{5},
\end{aligned}
$$

where $0<\theta<\min \left\{1,\left(2 d_{L} \wedge \delta \wedge 2 c\right) / 2 \sigma^{2}\right\}$. Select an appropriate constant $M>0$ to meet the following requirement:

$$
-M H+C \leq-2,
$$

where

$$
\begin{aligned}
C & =\sup _{(T, L, I, V) \in \mathbb{R}_{+}^{4}}\left\{-\frac{r}{2 T_{\max }} T^{2+\theta}-\frac{d_{L}}{2} L^{1+\theta}-\frac{\delta}{4} I^{1+\theta}\right. \\
& -\frac{c}{2}\left(\frac{V}{2 \bar{N}}\right)^{1+\theta}+\frac{r T}{T_{\max }}+E+d_{T}+d_{L}+a+\delta \\
& \left.+\frac{1}{2} \sigma_{1}^{2}+\frac{1}{2} \sigma_{2}^{2}+\frac{1}{2} \sigma_{3}^{2}\right\}<\infty .
\end{aligned}
$$

It is obvious to get that

$$
\liminf _{n \rightarrow \infty,(T, L, I, V) \in \mathbb{R}_{+}^{4} \backslash U_{n}} U(T, L, I, V)=+\infty,
$$

where $U_{n}=(1 / n, n) \times(1 / n, n) \times(1 / n, n) \times(1 / n, n)$ and $U(T, L, I, V)$ is a continuous function. So, $U(T, L, I, V)$ should have a minimum point $\left(\bar{T}_{0}, \bar{L}_{0}, \bar{I}_{0}, \bar{V}_{0}\right)$ in the interior of $\mathbb{R}_{+}^{4}$. Therefore, we formulate the following nonnegative $C^{2}$ function $\bar{U}: \mathbb{R}_{+}^{4} \rightarrow \mathbb{R}_{+}$:

$$
\bar{U}(T, L, I, V)=U(T, L, I, V)-U\left(\bar{T}_{0}, \bar{L}_{0}, \bar{I}_{0}, \bar{V}_{0}\right) .
$$

According to Itô formula,

$$
\begin{aligned}
& L U_{2}=-\frac{\lambda}{T}+d_{T}-r\left(1-\frac{T}{T_{\max }}\right)+\bar{k} V+\frac{1}{2} \sigma_{1}^{2}, \\
& L U_{3}=-\frac{\eta \bar{k} T V}{L}+\delta_{L}+a+\frac{1}{2} \sigma_{2}^{2}, \\
& L U_{4}=-\frac{(1-\eta) \bar{k} T V}{I}+\delta-a \frac{L}{I}+\frac{1}{2} \sigma_{3}^{2}, \\
& L U_{5}=\left(T+L+I+\frac{V}{2 \bar{N}}\right)^{\theta} \\
& \cdot\left[\lambda-d_{T} T+r T\left(1-\frac{T}{T_{\max }}\right)-d_{L} L-\frac{\delta I}{2}-\frac{c V}{2 \bar{N}}\right] \\
& +\frac{\theta}{2}\left(T+L+I+\frac{V}{2 \bar{N}}\right)^{\theta-1} \\
& \cdot\left[\sigma_{1}^{2} T^{2}+\sigma_{2}^{2} L^{2}+\sigma_{3}^{2} I^{2}+\sigma_{4}^{2}\left(\frac{V}{2 \bar{N}}\right)^{2}\right]
\end{aligned}
$$




$$
\begin{aligned}
& \leq\left(T+L+I+\frac{V}{2 \bar{N}}\right)^{\theta} \\
& \cdot\left(\lambda+r T-\frac{r T^{2}}{T_{\max }}-d_{L} L-\frac{\delta I}{2}-\frac{c V}{2 \bar{N}}\right) \\
& +\frac{\theta}{2}\left(\sigma_{1}^{2} \vee \sigma_{2}^{2} \vee \sigma_{3}^{2} \vee \sigma_{4}^{2}\right)\left(T+L+I+\frac{V}{2 \bar{N}}\right)^{\theta-1} \\
& \cdot\left[T^{2}+L^{2}+I^{2}+\left(\frac{V}{2 \bar{N}}\right)^{2}\right] \leq(\lambda+r T) \\
& \cdot\left(T+L+I+\frac{V}{2 \bar{N}}\right)^{\theta}-\frac{r}{T_{\max }} T^{2+\theta}-d_{L} L^{1+\theta}-\frac{\delta}{2} \\
& \cdot I^{1+\theta}-c\left(\frac{V}{2 \bar{N}}\right)^{1+\theta}+\frac{\theta}{2} \\
& \cdot \sigma^{2}\left[T^{1+\theta}+L^{1+\theta}+I^{1+\theta}+\left(\frac{V}{2 \bar{N}}\right)^{1+\theta}\right] \leq-\frac{r}{2 T_{\max }} \\
& \cdot T^{2+\theta}-\frac{d_{L}}{2} L^{1+\theta}-\frac{\delta}{4} I^{1+\theta}-\frac{c}{2}\left(\frac{V}{2 \bar{N}}\right)^{1+\theta}+E,
\end{aligned}
$$

where

$$
\begin{aligned}
E & =\sup _{(T, L, I, V) \in \mathbb{R}_{+}^{4}}\left\{-\frac{r}{2 T_{\max }} T^{2+\theta}-\frac{d_{L}}{2} L^{1+\theta}-\frac{\delta}{4} I^{1+\theta}\right. \\
& -\frac{c}{2}\left(\frac{V}{2 \bar{N}}\right)^{1+\theta} \\
& +\frac{\theta}{2} \sigma^{2}\left[T^{1+\theta}+L^{1+\theta}+I^{1+\theta}+\left(\frac{V}{2 \bar{N}}\right)^{1+\theta}\right] \\
& \left.+(\lambda+r T)\left(T+L+I+\frac{V}{2 \bar{N}}\right)^{\theta}\right\}<\infty .
\end{aligned}
$$

According to inequalities (24) and (31), we obtain that

$$
\begin{aligned}
L \bar{U} \leq & -M H+(M B+\bar{k}) V-\frac{\lambda}{T}+\frac{r T}{T_{\max }}-\frac{\eta \bar{k} T V}{L} \\
& -\frac{(1-\eta) \bar{k} V T}{I}-\frac{r}{2 T_{\max }} T^{2+\theta}-\frac{d_{L}}{2} L^{1+\theta} \\
& -\frac{\delta}{4} I^{1+\theta}-\frac{c}{2}\left(\frac{V}{2 \bar{N}}\right)^{1+\theta}+E+d_{T}+d_{L}+a+\delta \\
& +\frac{1}{2} \sigma_{1}^{2}+\frac{1}{2} \sigma_{2}^{2}+\frac{1}{2} \sigma_{3}^{2} .
\end{aligned}
$$

Next, we define a compact subset $D_{\varepsilon}$ which satisfies condition (A2) in Lemma A.1. Define the following bounded closed set:

$$
\begin{aligned}
D_{\varepsilon} & =\left\{\varepsilon \leq T \leq \frac{1}{\varepsilon}, \varepsilon^{3} \leq L \leq \frac{1}{\varepsilon^{3}}, \varepsilon^{3} \leq I \leq \frac{1}{\varepsilon^{3}}, \varepsilon \leq V\right. \\
& \left.\leq \frac{1}{\varepsilon}\right\},
\end{aligned}
$$

where $\varepsilon$ is a small enough constant. In set $\mathbb{R}_{+}^{4} \backslash D_{\varepsilon}$, we further select $\varepsilon$ sufficiently small which satisfies the following conditions:

$$
\begin{array}{r}
-M H+(M B+\bar{k}) \varepsilon+C \leq-1, \\
-\frac{\lambda}{\varepsilon}+F \leq-1, \\
-\frac{\eta \bar{k}}{\varepsilon}+F \leq-1, \\
-\frac{(1-\eta) \bar{k}}{\varepsilon}+F \leq-1, \\
-\frac{c}{4(2 N \varepsilon)^{1+\theta}}+F \leq-1, \\
-\frac{r}{4 \varepsilon^{2+\theta} T_{\max }}+F \leq-1, \\
-\frac{d_{L}}{4 \varepsilon^{3(1+\theta)}}+F \leq-1, \\
-\frac{\delta}{8 \varepsilon^{3(1+\theta)}}+F \leq-1,
\end{array}
$$

where

$$
\begin{aligned}
F & =\sup _{(T, L, I, V) \in \mathbb{R}_{+}^{4}}\left\{-\frac{r}{4 T_{\max }} T^{2+\theta}-\frac{d_{L}}{4} L^{1+\theta}-\frac{\delta}{8} I^{1+\theta}\right. \\
& -\frac{c}{4}\left(\frac{V}{2 \bar{N}}\right)^{1+\theta}+(M B+\bar{k}) V+\frac{r T}{T_{\max }}+E+d_{T} \\
& \left.+d_{L}+a+\delta+\frac{1}{2} \sigma_{1}^{2}+\frac{1}{2} \sigma_{2}^{2}+\frac{1}{2} \sigma_{3}^{2}\right\}<\infty .
\end{aligned}
$$

Set $\mathbb{R}_{+}^{4} \backslash D_{\varepsilon}$ is divided into the following eight domains:

$$
\begin{aligned}
& D_{1}=\left\{(T, L, I, V) \in \mathbb{R}_{+}^{4}, 0<V<\varepsilon\right\}, \\
& D_{2}=\left\{(T, L, I, V) \in \mathbb{R}_{+}^{4}, 0<T<\varepsilon\right\}, \\
& D_{3}=\left\{(T, L, I, V) \in \mathbb{R}_{+}^{4}, 0<L<\varepsilon^{3}, T \geq \varepsilon, V \geq \varepsilon\right\}, \\
& D_{4}=\left\{(T, L, I, V) \in \mathbb{R}_{+}^{4}, 0<I<\varepsilon^{3}, T \geq \varepsilon, V \geq \varepsilon\right\}, \\
& D_{5}=\left\{(T, L, I, V) \in \mathbb{R}_{+}^{4}, V>\frac{1}{\varepsilon}\right\}, \\
& D_{6}=\left\{(T, L, I, V) \in \mathbb{R}_{+}^{4}, T>\frac{1}{\varepsilon}\right\}, \\
& D_{7}=\left\{(T, L, I, V) \in \mathbb{R}_{+}^{4}, L>\frac{1}{\varepsilon^{3}}\right\}, \\
& D_{8}=\left\{(T, L, I, V) \in \mathbb{R}_{+}^{4}, I>\frac{1}{\varepsilon^{3}}\right\} .
\end{aligned}
$$

Obviously, $D_{\varepsilon}^{c}=D_{1} \cup D_{2} \cup D_{3} \cup D_{4} \cup D_{5} \cup D_{6} \cup D_{7} \cup D_{8}$. Case 1. If $(T, L, I, V) \in D_{1}$, 


$$
\begin{aligned}
L \bar{U} \leq & -M H+(M B+\bar{k}) V+\frac{r T}{T_{\max }}-\frac{r}{2 T_{\max }} T^{2+\theta} \\
& -\frac{d_{L}}{2} L^{1+\theta}-\frac{\delta}{4} I^{1+\theta}-\frac{c}{2}\left(\frac{V}{2 \bar{N}}\right)^{1+\theta}+E+d_{T} \\
& +d_{L}+a+\delta+\frac{1}{2} \sigma_{1}^{2}+\frac{1}{2} \sigma_{2}^{2}+\frac{1}{2} \sigma_{3}^{2} \\
\leq & -M H+(M B+\bar{k}) \varepsilon+C .
\end{aligned}
$$

Based on condition (35), it indicates that $L \bar{U} \leq-1$ for any $(T, L, I, V) \in D_{1}$.

Case 2. If $(T, L, I, V) \in D_{2}$,

$$
\begin{aligned}
L \bar{U} \leq & -\frac{\lambda}{T}+(M B+\bar{k}) V+\frac{r T}{T_{\max }}-\frac{r}{4 T_{\max }} T^{2+\theta} \\
& -\frac{d_{L}}{4} L^{1+\theta}-\frac{\delta}{8} I^{1+\theta}-\frac{c}{4}\left(\frac{V}{2 \bar{N}}\right)^{1+\theta}+E+d_{T} \\
& +d_{L}+a+\delta+\frac{1}{2} \sigma_{1}^{2}+\frac{1}{2} \sigma_{2}^{2}+\frac{1}{2} \sigma_{3}^{2} \leq-\frac{\lambda}{T}+F \\
\leq & -\frac{\lambda}{\varepsilon}+F .
\end{aligned}
$$

In view of (36), we get $L \bar{U} \leq-1$ for any $(T, L, I, V) \in D_{2}$.

Case 3. If $(T, L, I, V) \in D_{3}$,

$$
\begin{aligned}
L \bar{U} \leq & -\frac{\eta \bar{k} T V}{L}+(M B+\bar{k}) V+\frac{r T}{T_{\max }}-\frac{r}{4 T_{\max }} T^{2+\theta} \\
& -\frac{d_{L}}{4} L^{1+\theta}-\frac{\delta}{8} I^{1+\theta}-\frac{c}{4}\left(\frac{V}{2 \bar{N}}\right)^{1+\theta}+E+d_{T} \\
& +d_{L}+a+\delta+\frac{1}{2} \sigma_{1}^{2}+\frac{1}{2} \sigma_{2}^{2}+\frac{1}{2} \sigma_{3}^{2} \\
\leq & -\frac{\eta \bar{k} T V}{L}+F \leq-\frac{\eta \bar{k}}{\varepsilon}+F .
\end{aligned}
$$

According to (37), we obtain that $L \bar{U} \leq-1$ for any $(T, L$, $I, V) \in D_{3}$.

Case 4. If $(T, L, I, V) \in D_{4}$,

$$
\begin{aligned}
L \bar{U} \leq & -\frac{(1-\eta) \bar{k} V T}{I}+(M B+\bar{k}) V+\frac{r T}{T_{\max }} \\
& -\frac{r}{4 T_{\max }} T^{2+\theta}-\frac{d_{L}}{4} L^{1+\theta}-\frac{\delta}{8} I^{1+\theta} \\
& -\frac{c}{4}\left(\frac{V}{2 \bar{N}}\right)^{1+\theta}+E+d_{T}+d_{L}+a+\delta+\frac{1}{2} \sigma_{1}^{2} \\
& +\frac{1}{2} \sigma_{2}^{2}+\frac{1}{2} \sigma_{3}^{2} \leq-\frac{(1-\eta) \bar{k} V T}{I}+F \\
\leq & -\frac{(1-\eta) \bar{k}}{\varepsilon}+F .
\end{aligned}
$$

From condition (38), we deduce that $L \bar{U} \leq-1$ for any (T, $L$, $I, V) \in D_{4}$.

Case 5. If $(T, L, I, V) \in D_{5}$,

$$
\begin{aligned}
L \bar{U} \leq & -\frac{c}{2}\left(\frac{V}{2 \bar{N}}\right)^{1+\theta}+(M B+\bar{k}) V+\frac{r T}{T_{\max }} \\
& -\frac{r}{4 T_{\max }} T^{2+\theta}-\frac{d_{L}}{4} L^{1+\theta}-\frac{\delta}{8} I^{1+\theta}+E+d_{T} \\
& +d_{L}+a+\delta+\frac{1}{2} \sigma_{1}^{2}+\frac{1}{2} \sigma_{2}^{2}+\frac{1}{2} \sigma_{3}^{2} \\
\leq & -\frac{c}{4}\left(\frac{V}{2 N}\right)^{1+\theta}+F \leq-\frac{c}{4(2 N \varepsilon)^{1+\theta}}+F .
\end{aligned}
$$

In view of (39), we obtain that $L \bar{U} \leq-1$ for any $(T, L, I, V) \in$ $D_{5}$.

Case 6. If $(T, L, I, V) \in D_{6}$,

$$
\begin{aligned}
L \bar{U} \leq & -\frac{r}{2 T_{\max }} T^{2+\theta}+(M B+\bar{k}) V+\frac{r T}{T_{\max }}-\frac{d_{L}}{4} L^{1+\theta} \\
& -\frac{\delta}{8} I^{1+\theta}-\frac{c}{4}\left(\frac{V}{2 \bar{N}}\right)^{1+\theta}+E+d_{T}+d_{L}+a+\delta \\
& +\frac{1}{2} \sigma_{1}^{2}+\frac{1}{2} \sigma_{2}^{2}+\frac{1}{2} \sigma_{3}^{2} \leq-\frac{r}{4 T_{\max }} T^{2+\theta}+F \\
\leq & -\frac{r}{4 \varepsilon^{2+\theta} T_{\max }}+F .
\end{aligned}
$$

By condition (40), we conclude that $L \bar{U} \leq-1$ for all $(T, L, I, V) \in D_{6}$.

Case 7. If $(T, L, I, V) \in D_{7}$,

$$
\begin{aligned}
L \bar{U} \leq & -\frac{d_{L}}{2} L^{1+\theta}+(M B+\bar{k}) V+\frac{r T}{T_{\max }}-\frac{r}{4 T_{\max }} T^{2+\theta} \\
& -\frac{\delta}{8} I^{1+\theta}-\frac{c}{4}\left(\frac{V}{2 \bar{N}}\right)^{1+\theta}+E+d_{T}+d_{L}+a+\delta \\
& +\frac{1}{2} \sigma_{1}^{2}+\frac{1}{2} \sigma_{2}^{2}+\frac{1}{2} \sigma_{3}^{2} \leq-\frac{d_{L}}{4} L^{1+\theta}+F \\
\leq & -\frac{d_{L}}{4 \varepsilon^{3(1+\theta)}}+F .
\end{aligned}
$$

It derives that $L \bar{U} \leq-1$ for any $(T, L, I, V) \in D_{7}$ if condition (41) is satisfied.

Case 8. If $(T, L, I, V) \in D_{8}$,

$$
\begin{aligned}
L \bar{U} \leq & -\frac{\delta}{4} I^{1+\theta}+(M B+\bar{k}) V+\frac{r T}{T_{\max }}-\frac{r}{4 T_{\max }} T^{2+\theta} \\
& -\frac{d_{L}}{4} L^{1+\theta}-\frac{c}{4}\left(\frac{V}{2 \bar{N}}\right)^{1+\theta}+E+d_{T}+d_{L}+a
\end{aligned}
$$




$$
\begin{aligned}
& +\delta+\frac{1}{2} \sigma_{1}^{2}+\frac{1}{2} \sigma_{2}^{2}+\frac{1}{2} \sigma_{3}^{2} \leq-\frac{\delta}{8} I^{1+\theta}+F \\
\leq & -\frac{\delta}{8 \varepsilon^{3(1+\theta)}}+F .
\end{aligned}
$$

By condition (42), it is obvious to get that $L \bar{U} \leq-1$ for any $(T, L, I, V) \in D_{8}$. Clearly, from (45)-(52), there exists a sufficiently small $\varepsilon$, such that

$$
L \bar{U} \leq-1 \quad \forall(T, L, I, V) \in D_{\varepsilon}^{c} .
$$

By Lemma A.1 in the Appendix, we obtain that system (5) is ergodic and has a unique stationary distribution. This completes the proof.

Remark 3. For system (5), if there is no white noise, that is, $\sigma_{1}=\sigma_{2}=\sigma_{3}=\sigma_{4}=0$, then the critical condition $\mathscr{R}_{0}^{s}$ is the basic reproduction number $\mathscr{R}_{0}$ of its corresponding deterministic model (1). It indicates that the existence of the stationary distribution of our stochastic model is a generalization of its corresponding deterministic model to the stability of the infected equilibrium.

\section{Extinction}

Theorem 4. For any initial value $(T(0), L(0), I(0), V(0)) \in$ $\mathbb{R}_{+}^{4}$, one assumes $(T(t), L(t), I(t), V(t))$ be the solution of system (5). If $r-d_{T}>\sigma_{1}^{2} / 2$, then for almost $\omega \in \Omega$, the solution $(T(t), L(t), I(t), V(t))$ of system (5) is

$$
\begin{aligned}
& \limsup _{t \rightarrow \infty} \frac{1}{t} \ln \left(\frac{\sqrt{\mathscr{R}_{0}}}{d_{L}+a} L(t)+\frac{\sqrt{\mathscr{R}_{0}}}{a} I(t)+\frac{\mathscr{R}_{0}}{a \bar{N}} V(t)\right) \\
& \leq \phi, \quad \text { a.s. }
\end{aligned}
$$

where $\phi=\min \left\{d_{L}+a, \delta, c\right\}\left(\sqrt{\mathscr{R}_{0}}-1\right) \mathbf{I}_{\left\{\mathscr{R}_{0} \leq 1\right\}}+\max \left\{d_{L}+\right.$ $a, \delta, c\}\left(\sqrt{\mathscr{R}_{0}}-1\right) \mathbf{I}_{\left\{\mathscr{R}_{0}>1\right\}}+\left(c \sqrt{\mathscr{R}_{0}} / T_{0}\right) \int_{0}^{\infty}\left|x-T_{0}\right| \mu(x) d x-$ $(1 / 2)\left(\sigma_{2}^{-2}+\sigma_{3}^{-2}+\sigma_{4}^{-2}\right)^{-1}$, and the distribution of $T(t)$ converges weakly to a measure which has the invariant density

$$
\begin{array}{r}
\mu(x)=Q x^{-2+2\left(r-d_{T}\right) / \sigma_{1}^{2}} \exp \left\{-\frac{2}{\sigma_{1}^{2}}\left(\frac{\lambda}{x}+\frac{r x}{T_{\max }}\right)\right\}, \\
x \in(0, \infty),
\end{array}
$$

where $Q$ is a constant such that $\int_{0}^{\infty} \mu(x) d x=1$. If $\phi<0$, then $L, I$ and $V$ will be eliminated with probability one; namely,

$$
\begin{aligned}
& \lim _{t \rightarrow \infty} L(t)=0, \\
& \lim _{t \rightarrow \infty} I(t)=0, \\
& \lim _{t \rightarrow \infty} V(t)=0,
\end{aligned}
$$

a.s.

Proof. Firstly, we can obtain that the solution of system (5) is positive for any given positive initial value from Theorem 1 .
Consider the following auxiliary stochastic differential equation:

$$
\begin{aligned}
d x(t)= & {\left[\lambda-d_{T} x(t)+r x(t)\left(1-\frac{x(t)}{T_{\max }}\right)\right] d t } \\
& +\sigma_{1} x(t) d B_{1}(t) .
\end{aligned}
$$

For the initial value $x(0)=T(0)>0$ of system (57), denote $x(t)$ as its solution. Following the proof process in Theorem 3.1 of article [31], by the law of large numbers theorem [36], it is easy to get that $x(t)$ has the ergodic property with the invariant density

$$
\begin{array}{r}
\mu(x)=Q x^{-2+2\left(r-d_{T}\right) / \sigma_{1}^{2}} \exp \left\{-\frac{2}{\sigma_{1}^{2}}\left(\frac{\lambda}{x}+\frac{r x}{T_{\max }}\right)\right\}, \\
x \in(0, \infty),
\end{array}
$$

where $Q$ is a constant such that $\int_{0}^{\infty} \mu(x) d x=1$. It then follows that

$$
\lim _{t \rightarrow \infty} \frac{1}{t} \int_{0}^{t} x(s) d s=\int_{0}^{\infty} x \mu(x) d x \text { a.s. }
$$

By the comparison theorem of one-dimensional stochastic differential equation [37], we obtain that

$$
T(t) \leq x(t) \quad \forall t \geq 0 \text { a.s. }
$$

Next, we denote a matrix

$$
F=\left[\begin{array}{ccc}
0 & 0 & \frac{\eta \bar{k} T_{0}}{\left(d_{L}+a\right)} \\
\frac{a}{\delta} & 0 & \frac{(1-\eta) \bar{k} T_{0}}{\delta} \\
0 & \frac{\bar{N} \delta}{c} & 0
\end{array}\right] .
$$

It is easy to obtain that there must be a left eigenvector $\left(v_{1}, v_{2}, v_{3}\right)$ of matrix $F$ corresponding to the value $\sqrt{\mathscr{R}_{0}}[38]$, such that

$$
\left(v_{1}, v_{2}, v_{3}\right) F=\sqrt{\mathscr{R}_{0}}\left(v_{1}, v_{2}, v_{3}\right),
$$

where $\left(v_{1}, v_{2}, v_{3}\right)=\left(\sqrt{\mathscr{R}_{0}}, \delta \sqrt{\mathscr{R}_{0}} / a, c \mathscr{R}_{0} / a \bar{N}\right)$.

Define a $C^{2}$-function $G: \mathbb{R}_{+}^{4} \rightarrow \mathbb{R}$,

$$
G(L, I, V)=\alpha_{1} L+\alpha_{2} I+\alpha_{3} V
$$

where $\alpha_{1}=v_{1} /\left(d_{L}+a\right), \alpha_{2}=v_{2} / \delta, \alpha_{2}=v_{3} / c$. By applying Itô's formula, it yields

$$
\begin{gathered}
d(\ln G)=L(\ln G) d t+\frac{1}{G}\left[\alpha_{1} \sigma_{2} L d B_{2}(t)\right. \\
\left.+\alpha_{2} \sigma_{3} I d B_{3}(t)+\alpha_{3} \sigma_{4} V d B_{4}(t)\right]
\end{gathered}
$$


where

$$
\begin{aligned}
L(\ln G)= & \frac{\alpha_{1}}{G}\left(\eta \bar{k} T V-d_{L} L-a\right) \\
& +\frac{\alpha_{2}}{G}[(1-\eta) \bar{k} T V-\delta I+a L] \\
& +\frac{\alpha_{3}}{G}(\bar{N} \delta I-c V)-\frac{\alpha_{1}^{2} \sigma_{2}^{2} L^{2}}{2 G^{2}}-\frac{\alpha_{2}^{2} \sigma_{3}^{2} I^{2}}{2 G^{2}} \\
& -\frac{\alpha_{3}^{2} \sigma_{4}^{2} V^{2}}{2 G^{2}} \\
:= & J-\frac{\alpha_{1}^{2} \sigma_{2}^{2} L^{2}}{2 G^{2}}-\frac{\alpha_{2}^{2} \sigma_{3}^{2} I^{2}}{2 G^{2}}-\frac{\alpha_{3}^{2} \sigma_{4}^{2} V^{2}}{2 G^{2}} .
\end{aligned}
$$

We have

$$
\begin{aligned}
G^{2} & =\left(\alpha_{1} \sigma_{2} L \frac{1}{\sigma_{2}}+\alpha_{2} \sigma_{3} I \frac{1}{\sigma_{3}}+\alpha_{3} \sigma_{4} V \frac{1}{\sigma_{4}}\right)^{2} \\
& \leq\left(\alpha_{1}^{2} \sigma_{2}^{2} L^{2}+\alpha_{2}^{2} \sigma_{3}^{2} I^{2}+\alpha_{3}^{2} \sigma_{4}^{2} V^{2}\right)\left(\frac{1}{\sigma_{2}^{2}}+\frac{1}{\sigma_{3}^{2}}+\frac{1}{\sigma_{4}^{2}}\right) .
\end{aligned}
$$

Moreover, by using (60), we get

$$
\begin{aligned}
J & =\frac{\alpha_{1}}{G}\left(\eta \bar{k} T V-d_{L} L-a\right)+\frac{\alpha_{2}}{G}[(1-\eta) \bar{k} T V-\delta I \\
& +a L]+\frac{\alpha_{3}}{G}(\bar{N} \delta I-c V) \leq \frac{\alpha_{1}}{G} \eta \bar{k}\left(x-T_{0}\right) V \\
& +\frac{\alpha_{1}}{G}\left(\eta \bar{k} T_{0} V-d_{L} L-a\right)+\frac{\alpha_{2}}{G}(1-\eta) \bar{k}\left(x-T_{0}\right) V \\
& +\frac{\alpha_{2}}{G}\left[(1-\eta) \bar{k} T_{0} V-\delta I+a L\right]+\frac{\alpha_{3}}{G}(\bar{N} \delta I-c V) \\
& \leq \frac{\bar{k}}{\alpha_{3}}\left[\alpha_{1} \eta+\alpha_{2}(1-\eta)\right]\left|x-T_{0}\right| \\
& +\frac{1}{G}\left\{\frac{v_{1}}{d_{L}+a}\left(\eta \bar{k} T_{0} V-d_{L} L-a\right)\right. \\
& \left.+\frac{v_{2}}{\delta}[(1-\eta) \bar{k} x V-\delta I+a L]+\frac{v_{3}}{c}(\bar{N} \delta I-c V)\right\} \\
& =\frac{\bar{k}}{\alpha_{3}}\left[\alpha_{1} \eta+\alpha_{2}(1-\eta)\right]\left|x-T_{0}\right|+\frac{1}{G}\left(v_{1}, v_{2}, v_{3}\right) \\
& +\left[F(L, I, V)^{T}-(L, I, V)^{T}\right]=\frac{\bar{k}}{\alpha_{3}}\left[\alpha_{1} \eta\right. \\
& \left.+\alpha_{2}(1-\eta)\right]\left|x-T_{0}\right|+\frac{1}{G}\left(\sqrt{\mathscr{R}_{0}}-1\right)\left(v_{1} L+v_{2} I\right. \\
& +\frac{1}{G}\left(\sqrt{\alpha_{3}}\left[\alpha_{1} \eta+\alpha_{2}(1-\eta)\right]\left|x-T_{0}\right|\right. \\
& \bar{k} \\
&
\end{aligned}
$$

$$
\begin{aligned}
& +\min \left\{d_{L}+a, \delta, c\right\}\left(\sqrt{\mathscr{R}_{0}}-1\right) \mathbf{I}_{\left\{\mathscr{R}_{0} \leq 1\right\}} \\
& +\max \left\{d_{L}+a, \delta, c\right\}\left(\sqrt{\mathscr{R}_{0}}-1\right) \mathbf{I}_{\left\{\mathscr{R}_{0}>1\right\}} .
\end{aligned}
$$

By (66) and (67), we obtain that

$$
\begin{aligned}
L(\ln G) \leq & \min \left\{d_{L}+a, \delta, c\right\}\left(\sqrt{\mathscr{R}_{0}}-1\right) \mathbf{I}_{\left\{\mathscr{R}_{0} \leq 1\right\}} \\
& +\max \left\{d_{L}+a, \delta, c\right\}\left(\sqrt{\mathscr{R}_{0}}-1\right) \mathbf{I}_{\left\{\mathscr{R}_{0}>1\right\}} \\
& +\frac{\bar{k}}{\alpha_{3}}\left[\alpha_{1} \eta+\alpha_{2}(1-\eta)\right]\left|x-T_{0}\right| \\
& -\frac{1}{2}\left(\sigma_{2}^{-2}+\sigma_{3}^{-2}+\sigma_{4}^{-2}\right)^{-1} .
\end{aligned}
$$

From (64), we further derive that

$$
\begin{aligned}
& d(\ln G) \leq\left\{\min \left\{d_{L}+a, \delta, c\right\}\left(\sqrt{\mathscr{R}_{0}}-1\right) \mathbf{I}_{\left\{\mathscr{R}_{0} \leq 1\right\}}\right. \\
& +\max \left\{d_{L}+a, \delta, c\right\}\left(\sqrt{\mathscr{R}_{0}}-1\right) \mathbf{I}_{\left\{\mathscr{R}_{0}>1\right\}} \\
& +\frac{\bar{k}}{\alpha_{3}}\left[\alpha_{1} \eta+\alpha_{2}(1-\eta)\right]\left|x-T_{0}\right| \\
& \left.\quad-\frac{1}{2}\left(\sigma_{2}^{-2}+\sigma_{3}^{-2}+\sigma_{4}^{-2}\right)^{-1}\right\} d t \\
& +\frac{1}{G}\left[\alpha_{1} \sigma_{2} L d B_{2}(t)+\alpha_{2} \sigma_{3} I d B_{3}(t)\right. \\
& \left.+\alpha_{3} \sigma_{4} V d B_{4}(t)\right] .
\end{aligned}
$$

Integrate (69) from 0 to $t$, and divide by $t$ on both sides, then

$$
\begin{aligned}
\frac{\ln G(t)}{t} \leq & \frac{\ln G(0)}{t} \\
& +\min \left\{d_{L}+a, \delta, c\right\}\left(\sqrt{\mathscr{R}_{0}}-1\right) \mathbf{I}_{\left\{\mathscr{R}_{0} \leq 1\right\}} \\
& +\max \left\{d_{L}+a, \delta, c\right\} \times\left(\sqrt{\mathscr{R}_{0}}-1\right) \mathbf{I}_{\left\{\mathscr{R}_{0}>1\right\}} \\
& +\frac{\bar{k}\left[\alpha_{1} \eta+\alpha_{2}(1-\eta)\right]}{\alpha_{3} t} \int_{0}^{t}\left|x(s)-T_{0}\right| d s \\
& -\frac{1}{2}\left(\sigma_{2}^{-2}+\sigma_{3}^{-2}+\sigma_{4}^{-2}\right)^{-1} \\
& +\frac{1}{t} \int_{0}^{t} \frac{\alpha_{1} \sigma_{2} L(s)}{G(s)} d B_{2}(s) \\
& +\frac{1}{t} \int_{0}^{t} \frac{\alpha_{2} \sigma_{3} I(s)}{G(s)} d B_{3}(s) \\
& +\frac{1}{t} \int_{0}^{t} \frac{\alpha_{3} \sigma_{4} V(s)}{G(s)} d B_{4}(s)
\end{aligned}
$$




$$
\begin{aligned}
= & \frac{\ln G(0)}{t} \\
& +\min \left\{d_{L}+a, \delta, c\right\}\left(\sqrt{\mathscr{R}_{0}}-1\right) \mathbf{I}_{\left\{\mathscr{R}_{0} \leq 1\right\}} \\
& +\max \left\{d_{L}+a, \delta, c\right\} \times\left(\sqrt{\mathscr{R}_{0}}-1\right) \mathbf{I}_{\left\{\mathscr{R}_{0}>1\right\}} \\
& +\frac{\bar{k}\left[\alpha_{1} \eta+\alpha_{2}(1-\eta)\right]}{\alpha_{3} t} \int_{0}^{t}\left|x(s)-T_{0}\right| d s \\
& -\frac{1}{2}\left(\sigma_{2}^{-2}+\sigma_{3}^{-2}+\sigma_{4}^{-2}\right)^{-1}+\frac{M_{1}(t)}{t} \\
& +\frac{M_{2}(t)}{t}+\frac{M_{3}(t)}{t},
\end{aligned}
$$

where

$$
\begin{aligned}
& M_{1}(t)=\int_{0}^{t} \frac{\alpha_{1} \sigma_{2} L(s)}{G(s)} d B_{2}(s), \\
& M_{2}(t)=\int_{0}^{t} \frac{\alpha_{2} \sigma_{3} I(s)}{G(s)} d B_{3}(s), \\
& M_{3}(t)=\int_{0}^{t} \frac{\alpha_{3} \sigma_{4} V(s)}{G(s)} d B_{4}(s) .
\end{aligned}
$$

$M_{1}(t), M_{2}(t)$, and $M_{3}(t)$ are local martingales whose quadratic variations are $\left\langle M_{1}, M_{1}\right\rangle_{t}=\sigma_{2}^{2} \int_{0}^{t}\left(\alpha_{1} L(s) / G(s)\right) d s$ $\leq \sigma_{2}^{2} t,\left\langle M_{2}, M_{2}\right\rangle_{t}=\sigma_{3}^{2} \int_{0}^{t}\left(\alpha_{2} I(s) / G(s)\right) d s \leq \sigma_{3}^{2} t$, and $\left\langle M_{3}, M_{3}\right\rangle_{t}=\sigma_{4}^{2} \int_{0}^{t}\left(\alpha_{3} V(s) / G(s)\right) d s \leq \sigma_{4}^{2} t$. According to the strong law of large numbers for local martingale [33], we can obtain that

$$
\lim _{t \rightarrow \infty} \frac{M_{i}(t)}{t}=0 \quad \text { a.s., } i=1,2,3
$$

Because $x(t)$ is ergodic and $\int_{0}^{\infty} x \mu(x) d x<\infty$,

$$
\lim _{t \rightarrow \infty} \frac{1}{t} \int_{0}^{t}\left|x(s)-T_{0}\right| d s=\int_{0}^{\infty}\left|x-T_{0}\right| \mu(x) d x<\infty .
$$

Taking the superior limit on both sides of (70) and combining with (72) and (73) yield

$$
\begin{aligned}
\limsup _{t \rightarrow \infty} & \frac{\ln G(t)}{t} \\
\leq & \min \left\{d_{L}+a, \delta, c\right\}\left(\sqrt{\mathscr{R}_{0}}-1\right) \mathbf{I}_{\left\{\mathscr{R}_{0} \leq 1\right\}} \\
& +\max \left\{d_{L}+a, \delta, c\right\} \times\left(\sqrt{\mathscr{R}_{0}}-1\right) \mathbf{I}_{\left\{\mathscr{R}_{0}>1\right\}} \\
& +\frac{\bar{k}\left[\alpha_{1} \eta+\alpha_{2}(1-\eta)\right]}{\alpha_{3}} \lim _{t \rightarrow \infty} \frac{1}{t} \int_{0}^{t}\left|x(s)-T_{0}\right| d s \\
& -\frac{1}{2}\left(\sigma_{2}^{-2}+\sigma_{3}^{-2}+\sigma_{4}^{-2}\right)^{-1}
\end{aligned}
$$

$$
\begin{aligned}
= & \min \left\{d_{L}+a, \delta, c\right\}\left(\sqrt{\mathscr{R}_{0}}-1\right) \mathbf{I}_{\left\{\mathscr{R}_{0} \leq 1\right\}} \\
& +\max \left\{d_{L}+a, \delta, c\right\} \times\left(\sqrt{\mathscr{R}_{0}}-1\right) \mathbf{I}_{\left\{\mathscr{R}_{0}>1\right\}} \\
& +\frac{c \sqrt{\mathscr{R}_{0}}}{T_{0}} \int_{0}^{\infty}\left|x-T_{0}\right| \mu(x) d x \\
& -\frac{1}{2}\left(\sigma_{2}^{-2}+\sigma_{3}^{-2}+\sigma_{4}^{-2}\right)^{-1}:=\phi \quad \text { a.s. }
\end{aligned}
$$

which is the required assertion. In particular, if $\phi<0$, then it derives that

$$
\begin{aligned}
& \lim _{t \rightarrow \infty} \frac{\ln L(t)}{t}=0, \\
& \lim _{t \rightarrow \infty} \frac{\ln I(t)}{t}=0, \\
& \lim _{t \rightarrow \infty} \frac{\ln V(t)}{t}=0 \\
& \text { a.s.; }
\end{aligned}
$$

therefore, $\lim _{t \rightarrow \infty} L(t)=0, \lim _{t \rightarrow \infty} I(t)=0$, and $\lim _{t \rightarrow \infty} V(t)=0$ a.s. In other words, the populations $L, I$, and $V$ will tend to zero exponentially with probability one. This completes the proof.

\section{Numerical Simulations}

The numerical simulation is performed to investigate the effect of the white noises on model dynamical behavior. Applying Milstein's higher order method [39], system (5) can be rewritten as the following discrete form:

$$
\begin{aligned}
T_{m+1} & \\
= & T_{m} \\
& +\left[\lambda-d T_{m}-r T_{m}\left(1-\frac{T}{T_{\max }}\right)-\bar{k} V_{m} T_{m}\right] \Delta t \\
& +\sigma_{1} T_{m} \sqrt{\Delta t} \varphi_{1 m}+0.5 \sigma_{1}^{2} T_{m}\left(\Delta t \varphi_{1 m}^{2}-\Delta t\right), \\
& \quad+L_{m+1}+\left(\eta \bar{k} V_{m} T_{m}-d_{L} L_{m}+a L_{m}\right) \Delta t \\
& \quad{ }^{2} L_{m} \sqrt{\Delta t} \varphi_{2 m}+0.5 \sigma_{2}^{2} L_{m}\left(\Delta t \varphi_{2 m}^{2}-\Delta t\right), \\
& \quad+\left[(1-\eta) \bar{k} V_{m} T_{m}-\delta I_{m}+a L_{m}\right] \Delta t \\
I_{m+1} & I_{m}+\sigma_{3} I_{m} \sqrt{\Delta t} \varphi_{3 m}+0.5 \sigma_{3}^{2} I_{m}\left(\Delta t \varphi_{3 m}^{2}-\Delta t\right),
\end{aligned}
$$




$$
\begin{aligned}
& V_{m+1} \\
& \quad=V_{m}+\left(\bar{N} \delta T_{m}^{*}-c V_{m}\right) \Delta t+\sigma_{4} V_{m} \sqrt{\Delta t} \varphi_{4 m} \\
& \quad+0.5 \sigma_{4}^{2} V_{m}\left(\Delta t \varphi_{4 m}^{2}-\Delta t\right),
\end{aligned}
$$

where $\varphi_{1 m}, \varphi_{2 m}, \varphi_{3 m}$, and $\varphi_{4 m}, m=1,2, \ldots, n$, are the $m$ th realization of four independent Gaussian random variables with distribution $N(0,1)$, and the time step $\Delta t=0.01$.

In order to examine the effect of the white noises on model behavior numerically, we choose the same parameter values as the deterministic model (1). For the source of the parameter values, see literature [14] for detail.

$$
\begin{aligned}
\lambda & =10 \mu \mathrm{l}^{-1} \mathrm{day}^{-1}, \\
d_{T} & =0.03 \mathrm{day}^{-1}, \\
r & =0.1 \mathrm{day}^{-1} \\
T_{\max } & =1500 \mu \mathrm{l}^{-1}, \\
k & =0.0001 \mu \mathrm{l}^{-1} \mathrm{day}^{-1}, \\
\eta & =0.02, \\
d_{L} & =0.001 \text { day }^{-1}, \\
a & =0.1 \text { day }^{-1}, \\
\delta & =1 \text { day }^{-1}, \\
N & =1000 \text { virions } / \text { cell },^{-1} \\
c & =20 \text { day }^{-1}, \\
n_{r t} & =0.4, \\
n_{p} & =0.5,
\end{aligned}
$$

and the white noises $\sigma_{1}=\sigma_{2}=\sigma_{3}=0.08, \sigma_{4}=0.4$. For this set of parameters, we have the critical condition $\mathscr{R}_{0}^{s}=1.2726>1$, and system (5) has a unique stationary distribution (Theorem 2 is satisfied). For its corresponding deterministic model (1), the basic reproduction number $\mathscr{R}_{0}=1.7657>1$, and the unique infected equilibrium is locally asymptotically stable (result (II) in Section 2 is satisfied). When $\mathscr{R}_{0}^{s}>1$, Figure 1 shows that the unique stationary distribution occurs (see Figures 1(a) and 1(c)), and the corresponding distributions of total T-cells population (the sum of the healthy T-cell $T$, the latently infected T-cells $L$, and the actively infected T-cells $I$ ) and virus population are also given (see Figures 1(b) and 1(d)).

We further choose the white noises $\sigma_{1}=\sigma_{2}=\sigma_{3}=$ $0.3, \sigma_{4}=1.5$, and all the other parameters are the same as the above. We calculate that $\mathscr{R}_{0}^{s}=0.2524<1$, and Theorem 2 does not hold. In Figure 2, we can see that the virus can be eradicated for the stochastic model (5), while the virus is prevalent for the deterministic model (1). From Figures 1 and 2 , we examine that the existence of stationary distribution can be maintained if the white noises are small, and the virus can be eradicated if the white noises are big enough.

\section{Conclusions}

We have studied a stochastic HIV infection model with latent infection, target T-cells logistic growth, and antiretroviral therapy. The critical condition $\mathscr{R}_{0}^{s}$ of the stochastic model which is related to its corresponding deterministic model is derived. We obtain the existence of the unique stationary distribution when the critical condition is bigger than one. Moreover, the sufficient condition which is dependent on the basic reproduction number is obtained for the extinction of the virus. Numerically, we investigate that small fluctuations can be helpful to the persistence of the virus, while large fluctuations can be beneficial to the extinction of the virus.

For the special case, if the target T-cells logistic growth is not considered in our stochastic model, then system (5) can be rewritten as the following:

$$
\begin{aligned}
d T(t)= & {\left[\lambda-d_{T} T-\left(1-n_{r t}\right) k V T\right] d t+\sigma_{1} T d B_{1}(t), } \\
d L(t)= & {\left[\eta\left(1-n_{r t}\right) k V T-d_{L} L-a L\right] d t } \\
& +\sigma_{2} L d B_{2}(t), \\
d I(t)= & {\left[(1-\eta)\left(1-n_{r t}\right) k V T-\delta I+a L\right] d t } \\
& +\sigma_{3} I d B_{3}(t), \\
d V(t)= & {\left[\left(1-n_{p}\right) N \delta I-c V\right] d t+\sigma_{4} V d B_{4}(t) . }
\end{aligned}
$$

Model (78) is the corresponding stochastic model of the deterministic HIV basic latent infection model in literature [10-12], and our theoretical analysis results can also be established for this model. When there are no drug therapies in system (5), that is, $n_{r t}=n_{p}=0$, then our theoretical results are also satisfied.

In the process of virus infection, cytotoxic $\mathrm{T}$ lymphocyte (CTL) is the main immune cell in-host, which can limit the replication of virus in the body and determine the viral load $[2,40]$. Wang et al. in article [31] have considered the CTL immune response in HIV stochastic model; therefore, a stochastic HIV model with latent infection and CTL immune response will be our future work. In addition, under the influence of environment, the stochastic perturbation is not always treated as a constant but a periodic behavior [41]; thus HIV latent infection model regarding periodic perturbation is also our research work in the future.

\section{Appendix}

In order to prove the existence of the stationary distribution, we should introduce the theory of Has'minskii [35].

Let $X(t)$ be usual time-homogeneous Markov process in $\mathbb{R}^{l}$, and $X(t)$ satisfies the following stochastic differential equation:

$$
d X(t)=b(X) d t+\sum_{r=1}^{k} g_{r}(X) d B_{r}(t) .
$$




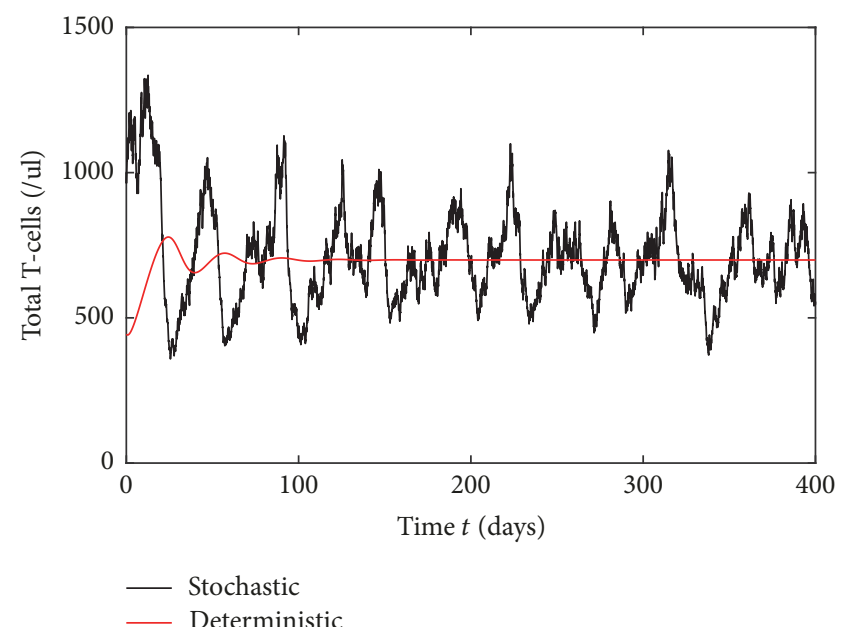

(a)

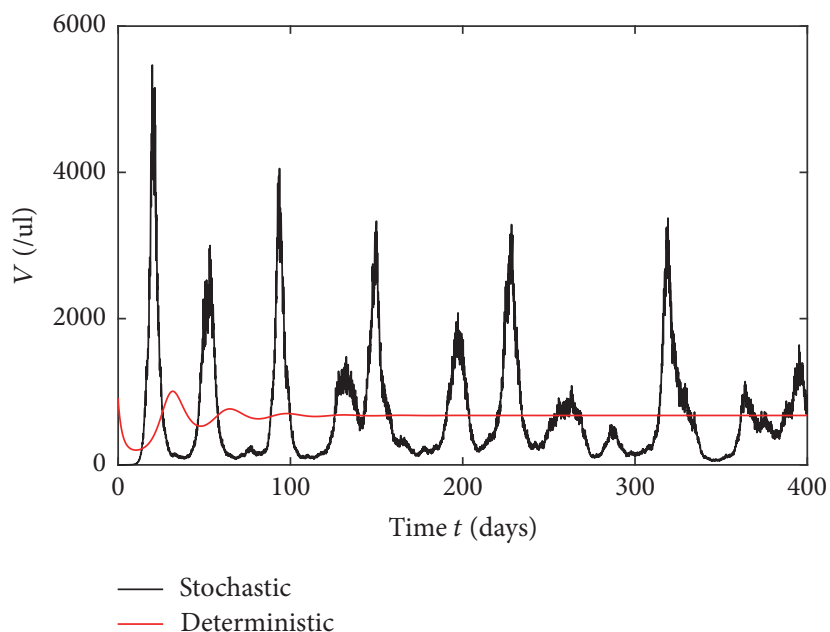

(c)

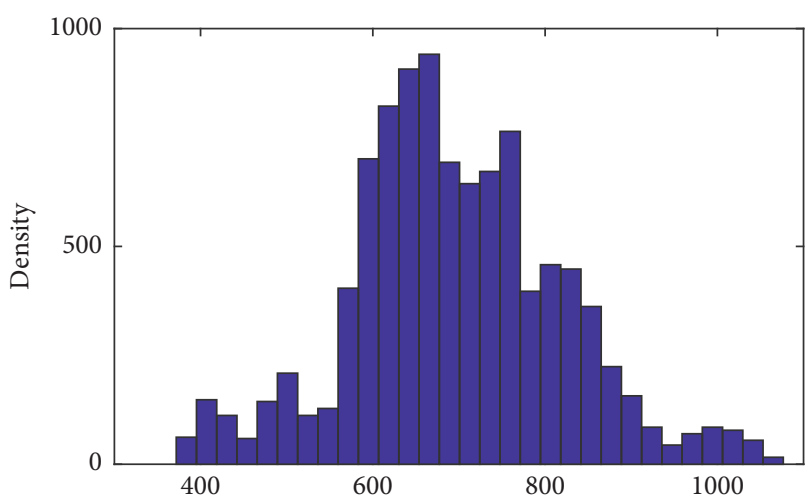

(b)

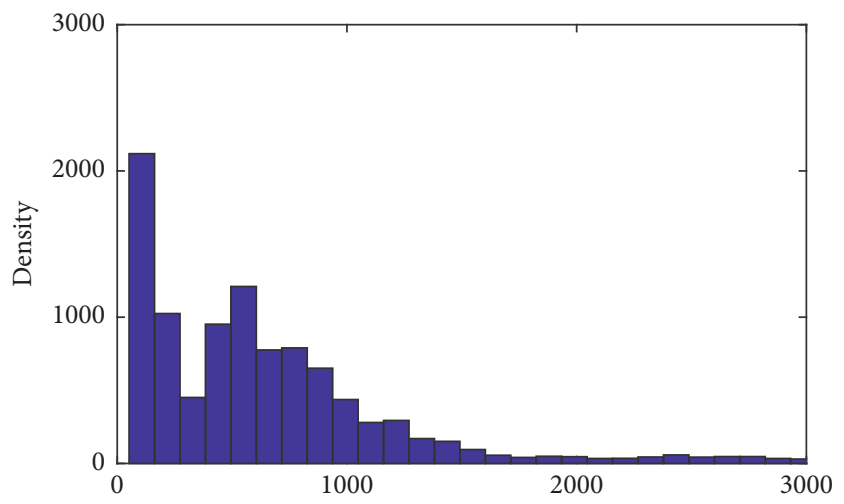

Figure 1: The solution of stochastic model (5) and its histogram with white noises $\sigma_{1}=\sigma_{2}=\sigma_{3}=0.08$ and $\sigma_{4}=0.4$. The black lines are the solutions of total T-cells and virus populations of stochastic model (5), and the red lines are the solutions of their deterministic model, respectively.

The definition of the diffusion matrix is as follows:

$$
A(x)=\left(a_{i j}(x)\right), \quad a_{i j}(x)=\sum_{r=1}^{k} g_{r}^{i}(x) g_{r}^{j}(x),
$$

where $X(t)$ is nonsingular.

Lemma A.1. It is assumed that there is a bounded open domain $U \subset \mathbb{R}^{l}$ with a regular boundary $\Gamma$, which has the following properties:

(A1) there is a positive number $E$ that makes $\sum_{i, j=1}^{l} a_{i j}(x) \xi_{i} \xi_{i} \geq R|\xi|^{2}$ satisfied, for $x \in U$ and $\xi \in \mathbb{R}^{l}$, where $R$ is a constant;

(A2) there is a nonnegative $C^{2}$-function $U$ that makes $L U$ negative for any $\mathbb{R}^{l} \backslash D$, then

$$
\mathbb{P}\left\{\lim _{t \rightarrow \infty} \frac{1}{t} \int_{0}^{t} f(X(s)) d s=\int_{\mathbb{R}}^{l} f(x) \pi(d x)\right\}=1
$$

for all $x \in \mathbb{R}^{l}$, where $\pi$ is the stationary distribution of the Markov process $X(t)$ and $f(x)$ is an integrable function of measure $\pi$. Then, the Markov process $X(t)$ has a unique ergodic stationary distribution $\pi(\cdot)$.

\section{Data Availability}

The data used to support the findings of this study are included within the article.

\section{Conflicts of Interest}

The authors declare that there are no conflicts of interest regarding the publication of this paper.

\section{Acknowledgments}

The authors are supported by National Natural Science Foundation of China (11401589, 11301543, 11501446, and 11101127), 


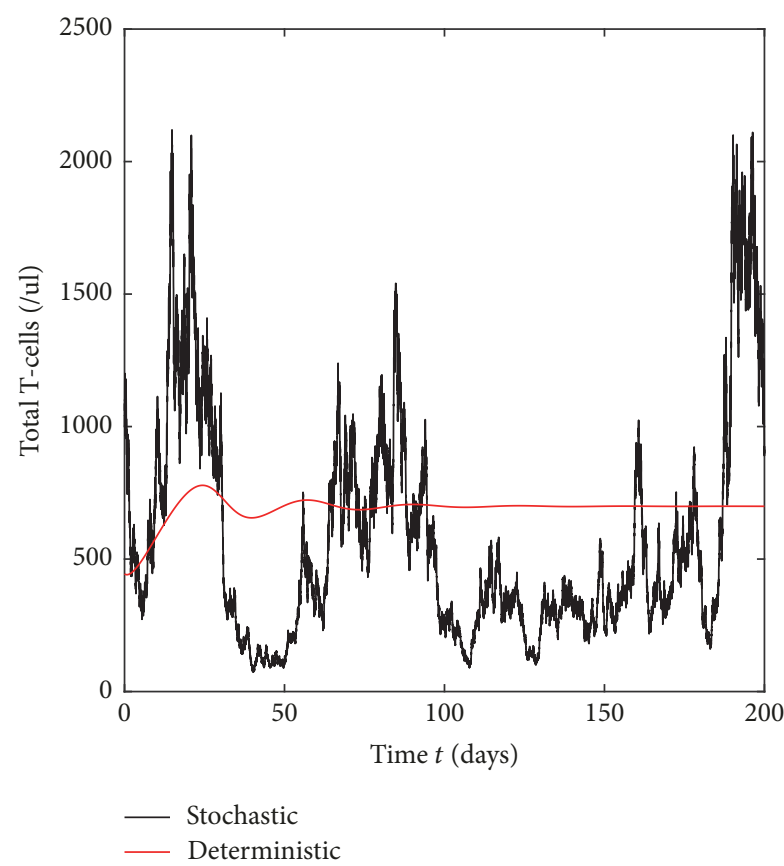

(a)

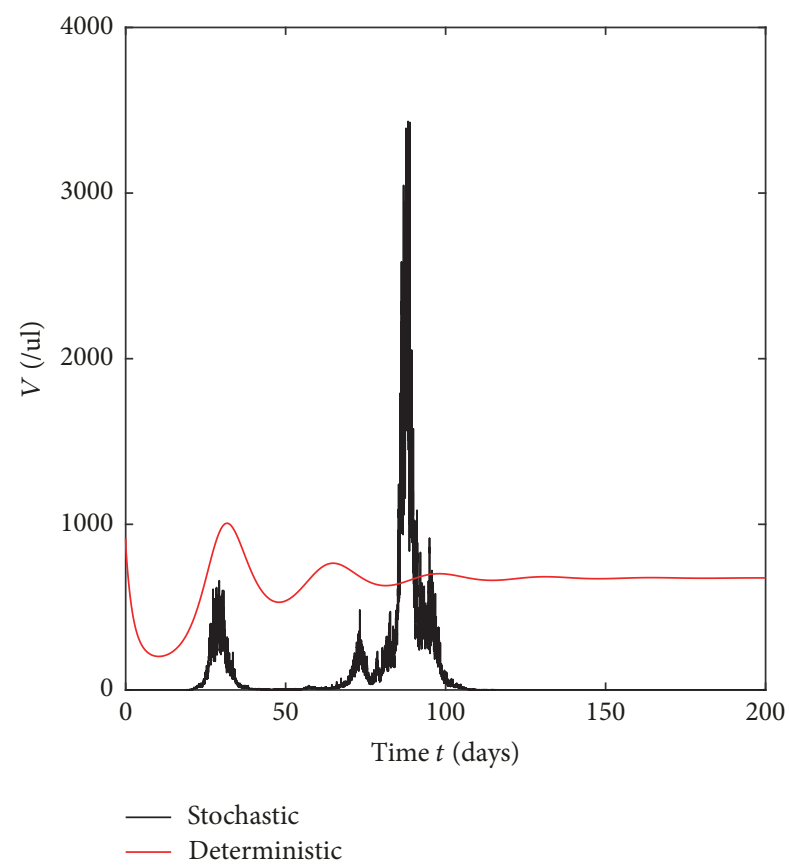

(b)

FIGURE 2: The virus can be eradicated for the stochastic model (5) when the white noises $\sigma_{1}=\sigma_{2}=\sigma_{3}=0.3$ and $\sigma_{4}=1.5$, while the virus is prevalent for its corresponding deterministic model.

the Fundamental Research Funds for the Central Universities (18CX02049A and 17CX02066), the Foundation for Leaders of Disciplines in Science of Haust (13490002), the Natural Science Research Fund of Northwest University (14NW17), and the Scientific Research Plan Projects of Education Department of Shaanxi Provincial Government (15JK1765).

\section{References}

[1] P. De Leenheer and H. L. Smith, "Virus dynamics: a global analysis," SIAM Journal on Applied Mathematics, vol. 63, no. 4, pp. 1313-1327, 2003.

[2] M. A. Nowak and R. M. May, Virus Dynamics: Mathematics Principles of Immunology and Virology, Oxford University Press, London, UK, 2000.

[3] A. S. Perelson and P. W. Nelson, "Mathematical analysis of HIV1 dynamics in vivo," SIAM Review, vol. 41, no. 1, pp. 3-44, 1999.

[4] N. M. Dixit and A. S. Perelson, "Complex patterns of viral load decay under antiretroviral therapy: influence of pharmacokinetics and intracellular delay," Journal of Theoretical Biology, vol. 226, no. 1, pp. 95-109, 2004.

[5] M. A. Nowak, S. Bonhoeffer, G. M. Shaw, and R. M. May, "Antiviral drug treatment: dynamics of resistance in free virus and infected cell populations," Journal of Theoretical Biology, vol. 184, no. 2, pp. 203-217, 1997.

[6] Y. Wang, Y. Zhou, J. Wu, and J. Heffernan, "Oscillatory viral dynamics in a delayed HIV pathogenesis model," Mathematical Biosciences, vol. 219, no. 2, pp. 104-112, 2009.

[7] Y. Wang, Y. Zhou, F. Brauer, and J. M. Heffernan, "Viral dynamics model with CTL immune response incorporating antiretroviral therapy," Journal of Mathematical Biology, vol. 67, no. 4, pp. 901-934, 2013.
[8] T.-W. Chun and A. S. Fauci, "Latent reservoirs of HIV: Obstacles to the eradication of virus," Proceedings of the National Acadamy of Sciences of the United States of America, vol. 96, no. 20, pp. 10958-10961, 1999.

[9] H. Kim and A. S. Perelson, "Viral and latent reservoir persistence in HIV-1-infected patients on therapy," PLoS Computational Biology, vol. 2, no. 10, pp. 1232-1247, 2006.

[10] L. Rong and A. S. Perelson, "Asymmetric division of activated latently infected cells may explain the decay kinetics of the HIV1 latent reservoir and intermittent viral blips," Mathematical Biosciences, vol. 217, no. 1, pp. 77-87, 2009.

[11] L. Rong and A. S. Perelson, "Modeling HIV persistence, the latent reservoir, and viral blips," Journal of Theoretical Biology, vol. 260, no. 2, pp. 308-331, 2009.

[12] L. Rong and A. S. Perelson, "Modeling latently infected cell activation: viral and latent reservoir persistence, and viral blips in HIV-infected patients on potent therapy," PLoS Computational Biology, vol. 5, no. 10, Article ID e1000533, e1000533, 18 pages, 2009.

[13] S. Pankavich, "The effects of latent infection on the dynamics of HIV," Differential Equations Dynamical Systems, vol. 24, no. 3, p. 23, 2013.

[14] Y. Wang, J. Liu, and L. Liu, "Viral dynamics of an HIV model with latent infection incorporating antiretroviral therapy," Advances in Difference Equations, Paper No. 225, 15 pages, 2016.

[15] X. Wang, S. Tang, X. Song, and L. Rong, "Mathematical analysis of an HIV latent infection model including both virus-to-cell infection and cell-to-cell transmission," Journal of Biological Dynamics, vol. 11, no. suppl. 2, pp. 455-483, 2017.

[16] M. Nijhuis, C. A. B. Boucher, P. Schipper, T. Leitner, R. Schuurman, and J. Albert, "Stochastic processes strongly influence 
HIV-1 evolution during suboptimal protease-inhibitor therapy," Proceedings of the National Acadamy of Sciences of the United States of America, vol. 95, no. 24, pp. 14441-14446, 1998.

[17] I. M. Rouzine, B. S. Razooky, and L. S. Weinberger, "Stochastic variability in HIV affects viral eradication," Proceedings of the National Acadamy of Sciences of the United States of America, vol. 111, no. 37, pp. 13251-13252, 2014.

[18] A. Singh, B. Razooky, C. D. Cox, M. L. Simpson, and L. S. Weinberger, "Transcriptional bursting from the HIV-1 promoter is a significant source of stochastic noise in HIV-1 gene expression," Biophysical Journal, vol. 98, no. 8, pp. L32-L34, 2010.

[19] L. S. Weinberger, J. C. Burnett, J. E. Toettcher, A. P. Arkin, and D. V. Schaffer, "Stochastic gene expression in a lentiviral positive-feedback loop: HIV-1 Tat fluctuations drive phenotypic diversity," Cell, vol. 122, no. 2, pp. 169-182, 2005.

[20] Y. Cai, Y. Kang, and W. Wang, "A stochastic SIRS epidemic model with nonlinear incidence rate," Applied Mathematics and Computation, vol. 305, pp. 221-240, 2017.

[21] X. Leng, T. Feng, and X. Meng, "Stochastic inequalities and applications to dynamics analysis of a novel SIVS epidemic model with jumps," Journal of Inequalities and Applications, Paper No. 138, 25 pages, 2017.

[22] D. Li, J. Cui, M. Liu, and S. Liu, "The evolutionary dynamics of stochastic epidemic model with nonlinear incidence rate," Bulletin of Mathematical Biology, vol. 77, no. 9, pp. 1705-1743, 2015.

[23] X. Z. Meng, S. N. Zhao, T. Feng, and T. H. Zhang, "Dynamics of a novel nonlinear stochastic SIS epidemic model with double epidemic hypothesis," Journal of Mathematical Analysis and Applications, vol. 433, no. 1, pp. 227-242, 2016.

[24] Z. Teng and L. Wang, "Persistence and extinction for a class of stochastic SIS epidemic models with nonlinear incidence rate," Physica A: Statistical Mechanics and its Applications, vol. 451, pp. 507-518, 2016.

[25] D. Zhao, T. Zhang, and S. Yuan, "The threshold of a stochastic SIVS epidemic model with nonlinear saturated incidence," Physica A: Statistical Mechanics and its Applications, vol. 443, pp. 372-379, 2016.

[26] N. Dalal, D. Greenhalgh, and X. Mao, "A stochastic model for internal HIV dynamics," Journal of Mathematical Analysis and Applications, vol. 341, no. 2, pp. 1084-1101, 2008.

[27] Z. Huang, Q. Yang, and J. Cao, "Complex dynamics in a stochastic internal HIV model," Chaos, Solitons \& Fractals, vol. 44, no. 11, pp. 954-963, 2011.

[28] H. C. Tuckwell and E. Le Corfec, "A stochastic model for early HIV-1 population dynamics," Journal of Theoretical Biology, vol. 195, no. 4, pp. 451-463, 1998.

[29] Y. Wang and D. Jiang, "Stationary distribution and extinction of a stochastic viral infection model," Discrete Dynamics in Nature and Society, Art. ID 6027509, 13 pages, 2017.

[30] D. Jiang, Q. Liu, N. Shi, T. Hayat, A. Alsaedi, and P. Xia, "Dynamics of a stochastic HIV-1 infection model with logistic growth," Physica A: Statistical Mechanics and its Applications, vol. 469, pp. 706-717, 2017.

[31] Y. Wang, D. Jiang, T. Hayat, and B. Ahmad, "A stochastic HIV infection model with T-cell proliferation and CTL immune response," Applied Mathematics and Computation, vol. 315, pp. 477-493, 2017.

[32] J. M. Conway and D. Coombs, "A stochastic model of latently infected cell reactivation and viral blip generation in treated HIV patients," PLoS Computational Biology, vol. 7, no. 4, Article ID e1002033, e1002033, 15 pages, 2011.
[33] X. Mao, Stochastic Differential Equations and Applications, Horwood, Chichester, UK, 2nd edition, 1997.

[34] X. Mao, G. Marion, and E. Renshaw, "Environmental Brownian noise suppresses explosions in population dynamics," Stochastic Processes and Their Applications, vol. 97, no. 1, pp. 95-110, 2002.

[35] R. Has'minskii, Stochastic Stability of Differential Equations, Sijthoff \& Noordhoff, Alphen aan den Rijn, The Netherlands, 1980.

[36] Y. A. Kutoyants, Statistical Inference for Ergodic Diffusion Processes, Springer, London, UK, 2004.

[37] N. Ikeda and S. Watanabe, "A comparison theorem for solutions of stochastic differential equations and its applications," Osaka Journal of Mathematics, vol. 14, no. 3, pp. 619-633, 1977.

[38] A. Berman and R. J. Plemmons, Nonnegative Matrices in Mathematical Sciences, Academic Press, New York, NY, USA, 1979.

[39] D. J. Higham, "An algorithmic introduction to numerical simulation of stochastic differential equations," SIAM Review, vol. 43, no. 3, pp. 525-546, 2001.

[40] M. A. Nowak and C. R. M. Bangham, "Population dynamics of immune responses to persistent viruses," Science, vol. 272, no. 5258, pp. 74-79, 1996.

[41] W. Wang, Y. Cai, J. Li, and Z. Gui, "Periodic behavior in a FIV model with seasonality as well as environment fluctuations," Journal of The Franklin Institute, vol. 354, no. 16, pp. 7410-7428, 2017. 


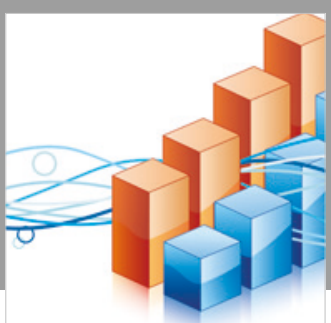

Advances in

Operations Research

\section{-n-m}
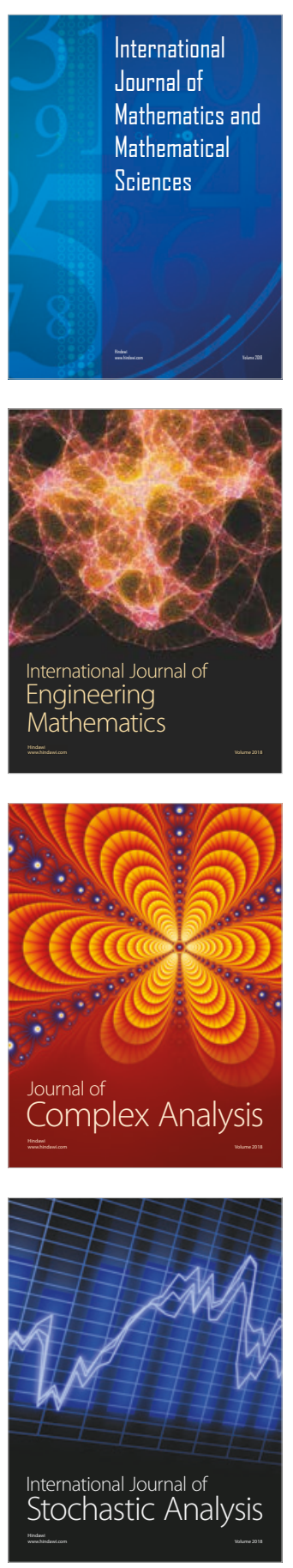
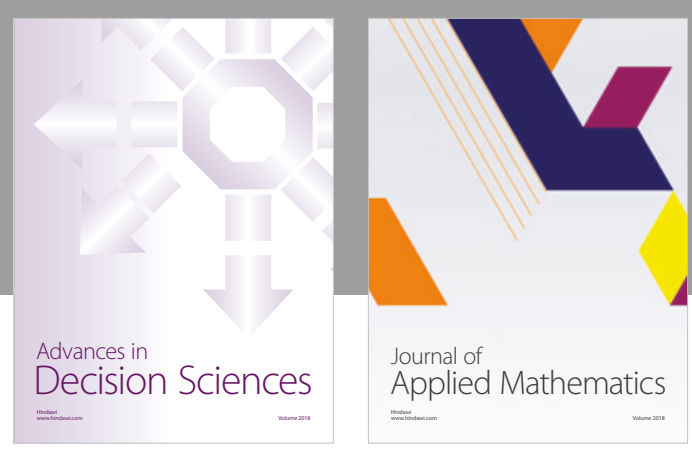

Journal of

Applied Mathematics
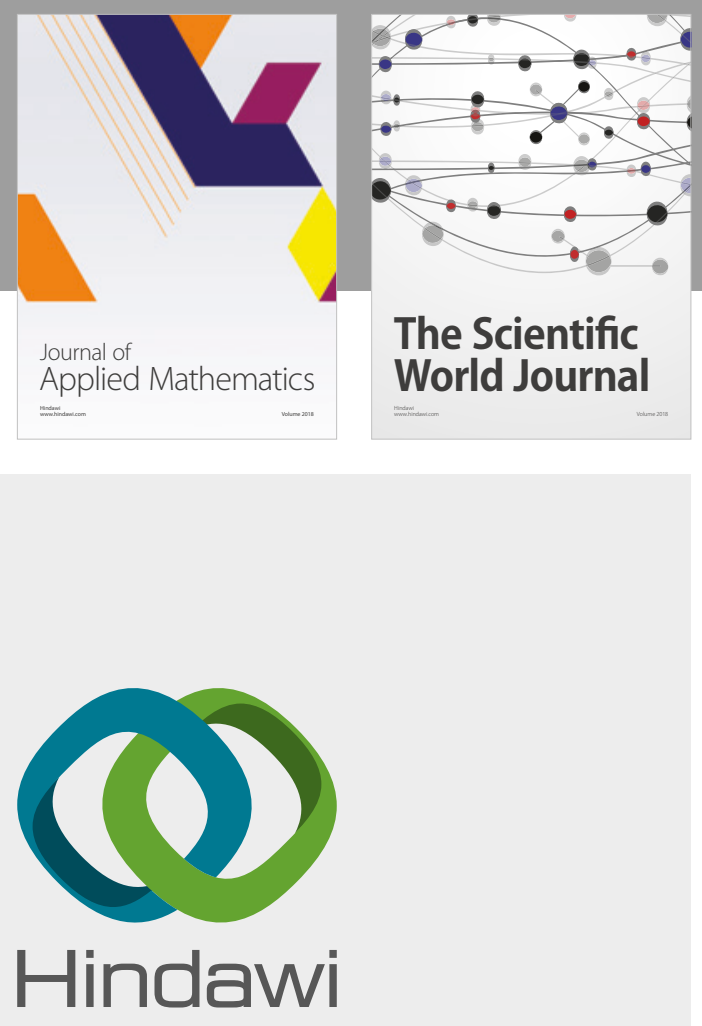

Submit your manuscripts at

www.hindawi.com

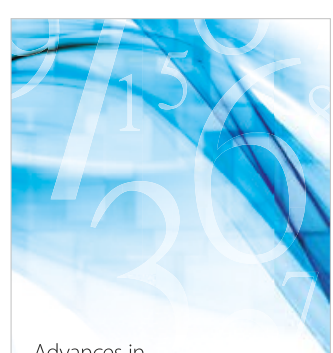

Advances in
Numerical Analysis
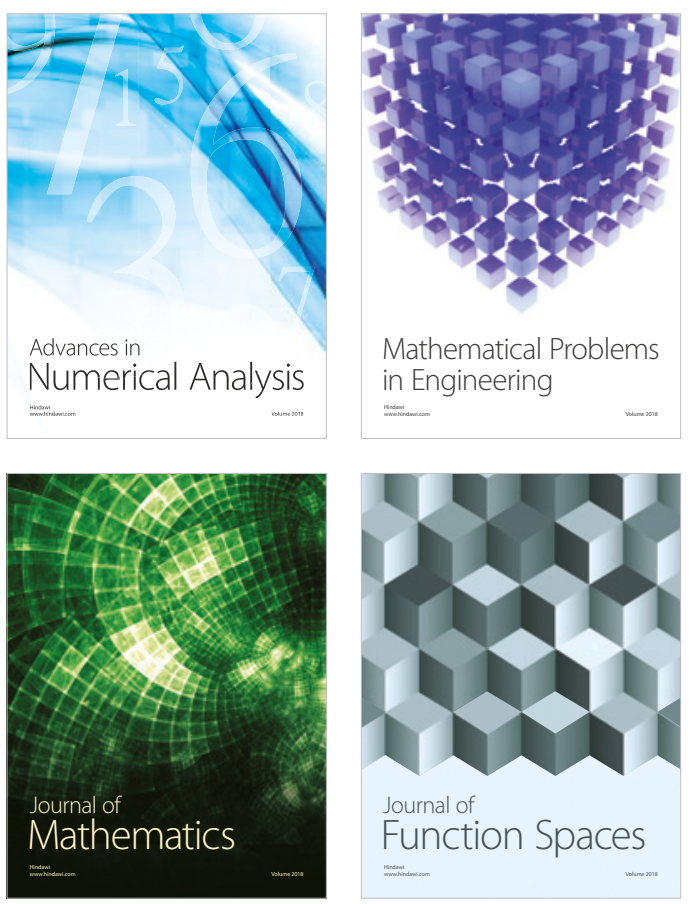

Mathematical Problems in Engineering

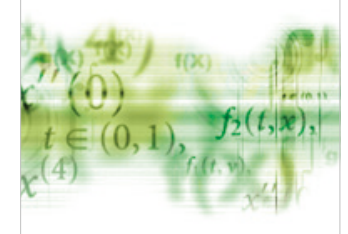

International Journal of

Differential Equations

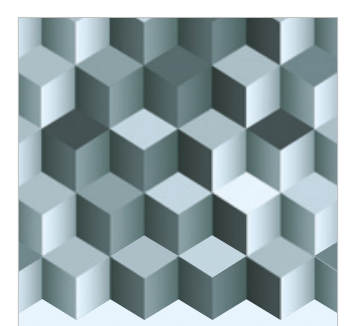

Journal of

Function Spaces

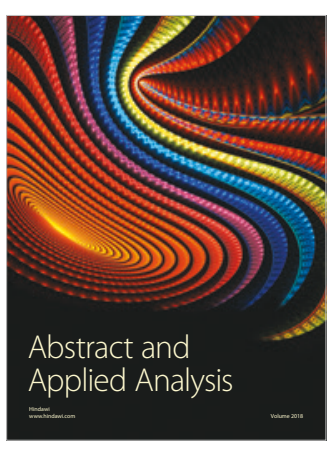

The Scientific

World Journal

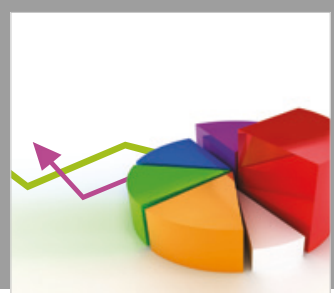

Journal of

Probability and Statistics
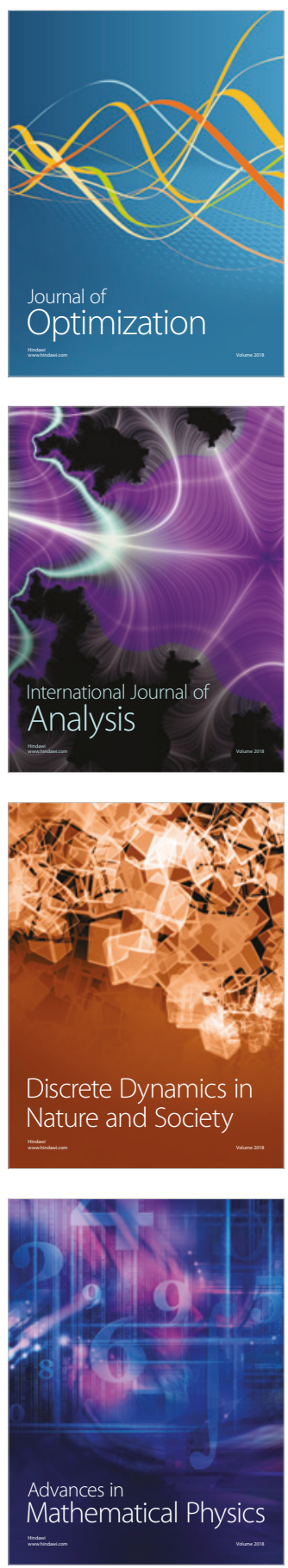